\title{
Quantum variant of Montgomery identity and Ostrowski-type inequalities for the mappings of two variables
}

\author{
Muhammad Aamir Ali ${ }^{1}$, Yu-Ming Chu ${ }^{2 *}$, Hüseyin Budak ${ }^{3}$, Abdullah Akkurt ${ }^{4}$, Hüseyin Yıldırım ${ }^{4}$ and \\ Manzoor Ahmed Zahid ${ }^{5}$
}

Correspondence:

chuyuming2005@126.com

2Department of Mathematics,

Huzhou University, Huzhou, China

Full list of author information is

available at the end of the article

\begin{abstract}
In this investigation, we demonstrate the quantum version of Montgomery identity for the functions of two variables. Then we use the result to derive some new Ostrowski-type inequalities for the functions of two variables via quantum integrals. We also consider the particular cases of the key results and offer some new integral inequalities.
\end{abstract}

Keywords: Ostrowski inequality; $q_{1} q_{2}$-integral; Quantum calculus; Coordinated convex function

\section{Introduction}

In the field of $q$-analysis, many studies have recently been carried out, starting with Euler owing to a vast requirement for mathematics that models quantum computing $q$-calculus occurred for the relationship between physics and mathematics. In different areas of mathematics, it has numerous applications such as combinatorics, number theory, basic hypergeometric functions, orthogonal polynomials, mechanics, the theory of relativity, and quantum theory $[1,2]$. Apparently, Euler invented this important mathematics branch. He used the $q$ parameter in Newton's work on infinite series. Later, in a methodical manner, the $q$-calculus without limit calculus was firstly given by Jackson [3]. In 1908-1909 the general form of the $q$-integral and $q$-difference operator was defined by Jackson [4]. In 1969, for the first time, Agarwal [5] defined the $q$-fractional derivative. In 1966-1967, Al-Salam [6] introduced a $q$-analog of the $q$-fractional integral and $q$-Riemann-Liouville fractional. In 2004, Rajkovic gave a definition of the Riemann-type $q$-integral, which was generalized to Jackson $q$-integral. In 2013, Tariboon [7] introduced the ${ }_{a} D_{q}$-difference operator. Recently, in 2020, Bermudo et al. [8] introduced the notions of the ${ }^{b} D_{q}$-derivative and integral.

Many well-known integral inequalities, such as the Hölder, Hermite-Hadamard, Simpson, Newton, Ostrowski, Cauchy-Bunyakovsky-Schwarz, Gruss, Gruss-Chebyshev, and other integral inequalities, have been studied in the setup of $q$-calculus using the concept of classical convexity. For more results in this direction, we refer to [9-20].

(c) The Author(s) 2021. This article is licensed under a Creative Commons Attribution 4.0 International License, which permits use sharing, adaptation, distribution and reproduction in any medium or format, as long as you give appropriate credit to the original author(s) and the source, provide a link to the Creative Commons licence, and indicate if changes were made. The images or other third party material in this article are included in the article's Creative Commons licence, unless indicated otherwise in a credit line to the material. If material is not included in the article's Creative Commons licence and your intended use is not permitted by statutory regulation or exceeds the permitted use, you will need to obtain permission directly from the copyright holder. To view a copy of this licence, visit http://creativecommons.org/licenses/by/4.0/. 
In 1938, Ostrowski [21] established the following interesting integral inequality.

Theorem 1 Let $F:[a, b] \rightarrow \mathbb{R}$ be a differentiable function on $(a, b)$ with bounded derivative, that is, $\left\|F^{\prime}\right\|_{\infty}:=\sup _{x \in(a, b)}\left|F^{\prime}(x)\right|<\infty$. Then we have the following integral inequality:

$$
\left|F(\tau)-\frac{1}{b-a} \int_{a}^{b} F(\tau) d \tau\right| \leq\left[\frac{1}{4}+\frac{\left(\tau-\frac{a+b}{2}\right)}{(b-a)^{2}}\right](b-a)\left\|F^{\prime}\right\|_{\infty}
$$

for all $\tau \in[a, b]$. The constant $\frac{1}{4}$ is the best possible.

Inequality (1.1) can be rewritten in the equivalent form

$$
\left|F(\tau)-\frac{1}{b-a} \int_{a}^{b} F(\tau) d \tau\right| \leq\left[\frac{(\tau-a)^{2}+(b-\tau)^{2}}{2(b-a)}\right]\left\|F^{\prime}\right\|_{\infty}
$$

Since 1938, when Ostrowski proved his famous inequality (see [21]), this inequality has been studied by many mathematicians in various fields, such as numerical analysis and probability.

Various generalizations and extensions of the Ostrowski integral inequality for boundedvariation, monotonic, Lipschitzian, convex, absolutely continuous, and $n$ times differentiable mappings with error estimates for some special means and some numerical quadrature rules were considered by many scientists. For more recent results, we refer to [22-32] and the references therein.

A formal definition of coordinated convex (concave) functions may be expressed as follows.

Definition 1 A function $F: \Delta \rightarrow \mathbb{R}$ is said to be coordinated convex on $\Delta$ if it satisfies the following inequality for all $(x, y),(z, w) \in \Delta$ and $\lambda, \mu \in[0,1]$ :

$$
\begin{aligned}
& F(\lambda x+(1-\lambda) z, \mu y+(1-\mu) w) \\
& \quad \leq \lambda \mu F(x, y)+\lambda(1-\mu) F(x, w)+\mu(1-\lambda) F(z, y)+(1-\lambda)(1-\mu) F(z, w) .
\end{aligned}
$$

The mapping $F$ is coordinated concave on $\Delta$ if inequality (1.3) holds in the reversed direction for all $(x, y),(z, w) \in \Delta$ and $\lambda, \mu \in[0,1]$.

Latif et al. [33] established the following Ostrowski-type inequalities for coordinated convex functions.

Theorem 2 Let $F: \Delta:=[a, b] \times[c, d] \rightarrow \mathbb{R}$ be a twice differentiable mapping on $\Delta^{\circ}$ with $a<b, c<d, a, c \geq 0$ such that $\frac{\partial^{2} F}{\partial s \partial t} \in L(\Delta)$. If $\left|\frac{\partial^{2} F}{\partial s \partial t}\right|$ is coordinated convex on $\Delta$ and $\left|\frac{\partial^{2} F}{\partial s \partial t}\right| \leq$ $M,(x, y) \in \Delta$, then we have the following inequality:

$$
\begin{array}{r}
\left|F(x, y)+\frac{1}{(b-a)(d-c)} \int_{a}^{b} \int_{c}^{d} F(t, s) d t d s-A_{1}\right| \\
\leq M\left[\frac{(x-a)^{2}+(b-x)^{2}}{2(b-a)}\right]\left[\frac{(y-c)^{2}+(d-y)^{2}}{2(d-c)}\right],
\end{array}
$$


where

$$
A_{1}=\frac{1}{d-c} \int_{c}^{d} F(x, s) d s+\frac{1}{b-a} \int_{a}^{b} F(t, y) d t .
$$

Theorem 3 Let $F: \Delta:=[a, b] \times[c, d] \rightarrow \mathbb{R}$ be a twice differentiable mapping on $\Delta^{\circ}$ with $a<b, c<d, a, c \geq 0$ such that $\frac{\partial^{2} F}{\partial s \partial t} \in L(\Delta)$. If $\left|\frac{\partial^{2} F}{\partial s \partial t}\right|^{p}$ is coordinated convex on $\Delta, p>1$, $\frac{1}{p}+\frac{1}{r}=1$, and $\left|\frac{\partial^{2} F}{\partial s \partial t}(x, y)\right| \leq M,(x, y) \in \Delta$, then we have the following inequality:

$$
\begin{aligned}
& \left|F(x, y)+\frac{1}{(b-a)(d-c)} \int_{a}^{b} \int_{c}^{d} F(t, s) d t d s-A_{1}\right| \\
& \quad \leq \frac{M}{(1+r)^{\frac{2}{r}}}\left[\frac{(x-a)^{2}+(b-x)^{2}}{2(b-a)}\right]\left[\frac{(y-c)^{2}+(d-y)^{2}}{2(d-c)}\right],
\end{aligned}
$$

where $A_{1}$ is defined in Theorem 2.

Theorem 4 Let $F: \Delta:=[a, b] \times[c, d] \rightarrow \mathbb{R}$ be a twice differentiable mapping on $\Delta^{\circ}$ with $a<b, c<d, a, c \geq 0$ such that $\frac{\partial^{2} F}{\partial s \partial t} \in L(\Delta)$. If $\left|\frac{\partial^{2} F}{\partial s \partial t}\right|^{p}$ is coordinated convex on $\Delta, p>1$, and $\left|\frac{\partial^{2} F}{\partial s \partial t}(x, y)\right| \leq M,(x, y) \in \Delta$, then we have the following inequality:

$$
\begin{array}{r}
\left|F(x, y)+\frac{1}{(b-a)(d-c)} \int_{a}^{b} \int_{c}^{d} F(t, s) d t d s-A_{1}\right| \\
\leq \frac{M}{4}\left[\frac{(x-a)^{2}+(b-x)^{2}}{2(b-a)}\right]\left[\frac{(y-c)^{2}+(d-y)^{2}}{2(d-c)}\right],
\end{array}
$$

where $A_{1}$ is defined in Theorem 2.

Inspired by this ongoing study, we establish some new quantum Ostrowski inequalities for $q_{1} q_{2}$-differentiable coordinated convex functions. This is the primary motivation of this paper. The ideas and strategies of the paper may open new venues for further research in this field.

\section{Preliminaries of $q$-calculus and some inequalities}

In this section, we review the basic notions and findings needed to prove our crucial results. Moreover, we use the following notation (see [34]):

$$
[n]_{q}=\frac{1-q^{n}}{1-q}=1+q+q^{2}+\cdots+q^{n-1}, \quad q \in(0,1) .
$$

Jackson [4] has defined the $q$-Jackson integral from 0 to $b$ for $0<q<1$ as follows:

$$
\int_{0}^{b} F(x) d_{q} x=(1-q) b \sum_{n=0}^{\infty} q^{n} F\left(b q^{n}\right),
$$

provided that the series converges absolutely.

Moreover, he defined the $q$-Jackson integral in a general interval $[a, b]$ as

$$
\int_{a}^{b} F(x) d_{q} x=\int_{0}^{b} F(x) d_{q} x-\int_{0}^{a} F(x) d_{q} x .
$$


Definition 2 ([35]) For a continuous function $F:[a, b] \rightarrow \mathbb{R}$, the $q_{a}$-derivative of $F$ at $x \in[a, b]$ is defined by the expression

$$
{ }_{a} D_{q} F(x)=\frac{F(x)-F(q x+(1-q) a)}{(1-q)(x-a)}, \quad x \neq a .
$$

The function $F$ is said to be $q_{a}$-differentiable on $[a, b]$ if ${ }_{a} D_{q} F(x)$ exists for all $x \in[a, b]$. If $a=0$ in (2.2), then ${ }_{0} D_{q} F(x)=D_{q} F(x)$, where $D_{q} F(x)$ is the familiar $q$-derivative of $F$ at $x \in[a, b]$ defined by the expression (see [34])

$$
D_{q} F(x)=\frac{F(x)-F(q x)}{(1-q) x}, \quad x \neq 0 .
$$

Definition 3 ([8]) For a continuous function $F:[a, b] \rightarrow \mathbb{R}$, the $q^{b}$-derivative of $F$ at $x \in$ $[a, b]$ is characterized by the expression

$$
{ }^{b} D_{q} F(x)=\frac{F(q x+(1-q) b)-F(x)}{(1-q)(b-x)}, \quad x \neq b .
$$

The function $F$ is said to be $q^{b}$-differentiable on $[a, b]$ if ${ }^{b} D_{q} F(x)$ exists for all $x \in[a, b]$. If $b=0$ in (2.2), then ${ }^{0} D_{q} F(x)=D_{q} F(x)$, where $D_{q} F(x)$ is the familiar $q$-derivative of $F$ at $x \in[a, b]$ defined by the expression (see [34])

$$
D_{q} F(x)=\frac{F(x)-F(q x)}{(1-q) x}, \quad x \neq 0 .
$$

Definition 4 ([35]) Let $F:[a, b] \rightarrow \mathbb{R}$ be a continuous function. Then the $q_{a}$-definite integral on $[a, b]$ is defined as

$$
\int_{a}^{b} F(x)_{a} d_{q} x=(1-q)(b-a) \sum_{n=0}^{\infty} q^{n} F\left(q^{n} b+\left(1-q^{n}\right) a\right)=(b-a) \int_{0}^{1} F((1-t) a+t b) d_{q} t .
$$

On the other hand, Bermudo et al. [8] gave the following new definition of the quantum integral.

Definition 5 Let $F:[a, b] \rightarrow \mathbb{R}$ be a continuous function. Then the $q^{b}$-definite integral on $[a, b]$ is defined as

$$
\int_{a}^{b} F(x)^{b} d_{q} x=(1-q)(b-a) \sum_{n=0}^{\infty} q^{n} F\left(q^{n} a+\left(1-q^{n}\right) b\right)=(b-a) \int_{0}^{1} F(t a+(1-t) b) d_{q} t .
$$

For more detail about $q^{b}$-integrals and corresponding inequalities, we refer to [8].

We have to give the following notation, which will be used many times in the next sections (see [34]):

$$
[n]_{q}=\frac{q^{n}-1}{q-1} .
$$


Lemma 1 ([36]) We have the equality

$$
\int_{a}^{b}(x-a)^{\alpha}{ }_{a} d_{q} x=\frac{(b-a)^{\alpha+1}}{[\alpha+1]_{q}}
$$

for $\alpha \in \mathbb{R} \backslash\{-1\}$.

Latif et al. [37] defined the $q_{a c}$-integral and partial $q$-derivatives for two-variable functions as follows.

Definition 6 Suppose that $F:[a, b] \times[c, d] \subset \mathbb{R}^{2} \rightarrow \mathbb{R}$ is a continuous function. Then the definite $q_{a c}$-integral on $[a, b] \times[c, d]$ is defined as

$$
\begin{aligned}
\int_{a}^{x} \int_{c}^{y} F(t, s){ }_{c} d_{q_{2}} s_{a} d_{q_{1}} t= & \left(1-q_{1}\right)\left(1-q_{2}\right)(x-a)(y-c) \\
& \times \sum_{n=0}^{\infty} \sum_{m=0}^{\infty} q_{1}^{n} q_{2}^{m} F\left(q_{1}^{n} x+\left(1-q_{1}^{n}\right) a, q_{2}^{m} y+\left(1-q_{2}^{m}\right) c\right)
\end{aligned}
$$

for $(x, y) \in[a, b] \times[c, d]$.

Lemma 2 If the assumptions of Definition 6 hold, then

$$
\begin{aligned}
\int_{y_{1}}^{y} \int_{x_{1}}^{x} F(t, s){ }_{a} d_{q_{1}} t_{c} d_{q_{2}} s & =\int_{y_{1}}^{y} \int_{a}^{x} F(t, s)_{a} d_{q_{1}} t_{c} d_{q_{2}} s-\int_{y_{1}}^{y} \int_{a}^{x_{1}} F(t, s)_{a} d_{q_{1}} t_{c} d_{q_{2}} s \\
= & \int_{c}^{y} \int_{a}^{x} F(t, s)_{a} d_{q_{1}} t_{c} d_{q_{2}} s-\int_{c}^{y_{1}} \int_{a}^{x} F(t, s)_{a} d_{q_{1}} t_{c} d_{q_{2}} s \\
& -\int_{c}^{y} \int_{a}^{x_{1}} F(t, s)_{a} d_{q_{1}} t_{c} d_{q_{2}} s+\int_{c}^{y_{1}} \int_{a}^{x_{1}} F(t, s)_{a} d_{q_{1}} t_{c} d_{q_{2}} s .
\end{aligned}
$$

Definition 7 ([37]) Let $F:[a, b] \times[c, d] \subseteq \mathbb{R}^{2} \rightarrow \mathbb{R}$ be a continuous function of two variables. Then the partial $q_{1}$-derivatives, $q_{2}$-derivatives, and $q_{1} q_{2}$-derivatives at $(x, y) \in$ $[a, b] \times[c, d]$ can be given as follows:

$$
\begin{aligned}
& \frac{{ }_{a} \partial_{q_{1}} F(x, y)}{{ }_{a} \partial_{q_{1}} x}=\frac{F\left(q_{1} x+\left(1-q_{1}\right) a, y\right)-F(x, y)}{\left(1-q_{1}\right)(x-a)}, \quad x \neq b, \\
& \begin{aligned}
& \frac{\partial_{q_{1}} F(x, y)}{{ }_{c} \partial_{q_{2}} y}=\frac{F\left(x, q_{2} y+\left(1-q_{2}\right) c\right)-F(x, y)}{\left(1-q_{2}\right)(y-c)}, \quad y \neq c, \\
& \frac{a_{a, c} \partial_{q_{1}, q_{2}}^{2} F(x, y)}{{ }_{a} \partial_{q_{1}} x_{c} \partial_{q_{2}} y}= \frac{1}{(x-a)(y-c)\left(1-q_{1}\right)\left(1-q_{2}\right)}\left[F\left(q_{1} x+\left(1-q_{1}\right) a, q_{2} y+\left(1-q_{2}\right) c\right)\right. \\
&\left.-F\left(q_{1} x+\left(1-q_{1}\right) a, y\right)-F\left(x, q_{2} y+\left(1-q_{2}\right) c\right)+F(x, y)\right], \quad x \neq a, y \neq c .
\end{aligned}
\end{aligned}
$$

For more detail on the related to $q$-integrals and derivatives for the functions of two variables, we refer to [37].

On the other hand, Budak et al. [38] gave the following definitions of $q_{a}^{d^{-}}, q_{b}^{c}$, and $q^{b d_{-}}$ integrals. 
Definition 8 Suppose that $F:[a, b] \times[c, d] \subset \mathbb{R}^{2} \rightarrow \mathbb{R}$ is continuous function. Then the following $q_{a}^{d}-, q_{c}^{b}$, and $q^{b d}$-integrals on $[a, b] \times[c, d]$ are defined by

$$
\begin{aligned}
\int_{a}^{x} \int_{y}^{d} F(t, s)^{d} d_{q_{2}} s_{a} d_{q_{1}} t= & \left(1-q_{1}\right)\left(1-q_{2}\right)(x-a)(d-y) \\
& \times \sum_{n=0}^{\infty} \sum_{m=0}^{\infty} q_{1}^{n} q_{2}^{m} F\left(q_{1}^{n} x+\left(1-q_{1}^{n}\right) a, q_{2}^{m} y+\left(1-q_{2}^{m}\right) d\right), \\
\int_{x}^{b} \int_{c}^{y} F(t, s){ }_{c} d_{q_{2}} s^{b} d_{q_{1}} t= & \left(1-q_{1}\right)\left(1-q_{2}\right)(b-x)(y-c) \\
& \times \sum_{n=0}^{\infty} \sum_{m=0}^{\infty} q_{1}^{n} q_{2}^{m} F\left(q_{1}^{n} x+\left(1-q_{1}^{n}\right) b, q_{2}^{m} y+\left(1-q_{2}^{m}\right) c\right),
\end{aligned}
$$

and

$$
\begin{aligned}
\int_{x}^{b} \int_{y}^{d} F(t, s)^{d} d_{q_{2}} s^{b} d_{q_{1}} t= & \left(1-q_{1}\right)\left(1-q_{2}\right)(b-x)(d-y) \\
& \times \sum_{n=0}^{\infty} \sum_{m=0}^{\infty} q_{1}^{n} q_{2}^{m} F\left(q_{1}^{n} x+\left(1-q_{1}^{n}\right) b, q_{2}^{m} y+\left(1-q_{2}^{m}\right) d\right),
\end{aligned}
$$

respectively, for $(x, y) \in[a, b] \times[c, d]$.

Definition 9 ([39]) Let $F:[a, b] \times[c, d] \subseteq \mathbb{R}^{2} \rightarrow \mathbb{R}$ be a continuous function of two variables. Then the partial $q_{1}$-derivatives, $q_{2}$-derivatives, and $q_{1} q_{2}$-derivatives at $(x, y) \in$ $[a, b] \times[c, d]$ can be given as follows:

$$
\begin{aligned}
& \frac{{ }^{b} \partial_{q_{1}} F(x, y)}{{ }^{b} \partial_{q_{1}} x}=\frac{F\left(q_{1} x+\left(1-q_{1}\right) b, y\right)-F(x, y)}{\left(1-q_{1}\right)(b-x)}, \quad x \neq b, \\
& \frac{d \partial_{q_{1}} F(x, y)}{{ }^{b} \partial_{q_{2}} y}=\frac{F\left(x, q_{2} y+\left(1-q_{2}\right) d\right)-F(x, y)}{\left(1-q_{2}\right)(d-y)}, \quad d \neq y, \\
& \frac{{ }_{a}^{d} \partial_{q_{1}, q_{2}}^{2} F(x, y)}{{ }_{a} \partial_{q_{1}} x d \partial_{q_{2}} y}=\frac{1}{(x-a)(d-y)\left(1-q_{1}\right)\left(1-q_{2}\right)}\left[F\left(q_{1} x+\left(1-q_{1}\right) a, q_{2} y+\left(1-q_{2}\right) d\right)\right. \\
& \left.-F\left(q_{1} x+\left(1-q_{1}\right) a, y\right)-F\left(x, q_{2} y+\left(1-q_{2}\right) d\right)+F(x, y)\right], \quad x \neq a, y \neq d, \\
& \frac{{ }_{c}^{b} \partial_{q_{1}, q_{2}}^{2} F(x, y)}{{ }^{b} \partial_{q_{1}} x_{c} \partial_{q_{2}} y}=\frac{1}{(b-x)(y-c)\left(1-q_{1}\right)\left(1-q_{2}\right)}\left[F\left(q_{1} x+\left(1-q_{1}\right) b, q_{2} y+\left(1-q_{2}\right) c\right)\right. \\
& \left.-F\left(q_{1} x+\left(1-q_{1}\right) b, y\right)-F\left(x, q_{2} y+\left(1-q_{2}\right) c\right)+F(x, y)\right], \quad x \neq b, y \neq c, \\
& \frac{{ }^{b, d} \partial_{q_{1}, q_{2}}^{2} F(x, y)}{{ }^{b} \partial_{q_{1}} x^{d} \partial_{q_{2}} y}=\frac{1}{(b-x)(d-y)\left(1-q_{1}\right)\left(1-q_{2}\right)}\left[F\left(q_{1} x+\left(1-q_{1}\right) b, q_{2} y+\left(1-q_{2}\right) d\right)\right. \\
& \left.-F\left(q_{1} x+\left(1-q_{1}\right) b, y\right)-F\left(x, q_{2} y+\left(1-q_{2}\right) d\right)+F(x, y)\right], \\
& x \neq b, y \neq d \text {. }
\end{aligned}
$$

3 Quantum Montgomery identity for the functions of two variables

In this section, we prove a quantum Montgomery identity via newly defined quantum integrals for functions of two variables. 
Lemma 3 Let $F: \Delta \subseteq \mathbb{R}^{2} \rightarrow \mathbb{R}$ be a twice $q_{1} q_{2}$-differentiable function on $\Delta^{\circ}$. If the partial $q_{1} q_{2}$-derivatives $\frac{b, d \partial_{q_{1}, q_{2}}^{2} F(t, s)}{{ }_{\partial} \partial_{q_{1}}{ }^{d} \partial_{q_{2}} s}$ are continuous and integrable on $[a, b] \times[c, d] \subseteq \Delta^{\circ}$, then we have the following identity for $q_{1} q_{2}$-integrals:

$$
\begin{aligned}
& \frac{1}{(b-a)(d-c)} \int_{a}^{b} \int_{c}^{d} F(t, s)^{b} d_{q_{1}} t^{d} d_{q_{2}} s-\frac{1}{b-a} \int_{a}^{b} F(t, y)^{b} d_{q_{1}} t \\
& \quad-\frac{1}{d-c} \int_{c}^{d} F(x, s)^{d} d_{q_{2}} s+F(x, y) \\
& =(b-a)(d-c) \int_{0}^{1} \int_{0}^{1} \Psi_{q_{1}}(t) \Psi_{q_{2}}(s) \frac{b^{b, d} \partial_{q_{1}, q_{2}}^{2} F(t a+(1-t) b, s c+(1-s) d)}{{ }^{b} \partial_{q_{1}} t^{d} \partial_{q_{2}} s} d_{q_{1}} t d_{q_{2}} s
\end{aligned}
$$

where

$$
\Psi_{q_{1}}(t)= \begin{cases}q_{1} t, & t \in\left[0, \frac{b-x}{b-a}\right), \\ q_{1} t-1, & t \in\left[\frac{b-x}{b-a}, 1\right],\end{cases}
$$

and

$$
\Psi_{q_{2}}(s)= \begin{cases}q_{2} s, & s \in\left[0, \frac{d-y}{d-c}\right), \\ q_{2} s-1, & s \in\left[\frac{d-y}{d-c}, 1\right]\end{cases}
$$

for $q_{1}, q_{2} \in(0,1)$.

Proof By Lemma 2 and the definitions of $\Psi_{q_{1}}(t)$ and $\Psi_{q_{2}}(s)$ we obtain

$$
\begin{aligned}
& \int_{0}^{1} \int_{0}^{1} \Psi_{q_{1}}(t) \Psi_{q_{2}}(s) \frac{b, d}{\partial_{q_{1}, q_{2}}^{2} F(t a+(1-t) b, s c+(1-s) d)} d_{q_{1}} t d_{q_{2}} s \\
& =\int_{0}^{\frac{b-x}{b-a}} \int_{0}^{\frac{d-y}{d-c}} \frac{b, d}{\partial_{q_{1}, q_{2}}^{2} F(t a+(1-t) b, s c+(1-s) d)} d_{q_{1}} t d_{q_{2}} s \\
& +\int_{0}^{\frac{b-x}{b-a}} \int_{0}^{1}\left(q_{2} s-1\right) \frac{b, d \partial_{q_{1}, q_{2}}^{2} F(t a+(1-t) b, s c+(1-s) d)}{b \partial_{q_{1}} t^{d} \partial_{q_{2}} s} d_{q_{2}} s \\
& +\int_{0}^{1} \int_{0}^{\frac{d-y}{d-c}}\left(q_{1} t-1\right) \frac{b, d \partial_{q_{1}, q_{2}}^{2} F(t a+(1-t) b, s c+(1-s) d)}{{ }^{b} \partial_{q_{1}} t^{d} \partial_{q_{2}} s} d_{q_{1}} t \\
& +\int_{0}^{1} \int_{0}^{1}\left(q_{2} s-1\right)\left(q_{1} t-s\right) \frac{b, d \partial_{q_{1}, q_{2}}^{2} F(t a+(1-t) b, s c+(1-s) d)}{{ }^{b} \partial_{q_{1}} t^{d} \partial_{q_{2}} s} d_{q_{1}} t d_{q_{2}} s \\
& =I_{1}+I_{2}+I_{3}+I_{4} \text {. }
\end{aligned}
$$

From Definition 9 we have

$$
\begin{aligned}
& \frac{b, d}{{ }^{b}{ }_{q_{1}, q_{2}}^{2} F(t a+(1-t) b, s c+(1-s) d)} \\
& \quad=\frac{1}{\left(1-q_{1}\right)\left(1-q_{2}\right)(b-x)(d-y) t s}\left[F\left(t q_{1} a+\left(1-t \partial_{1}\right) b, s q_{2} c+\left(1-s q_{2}\right) d\right)\right.
\end{aligned}
$$




$$
\begin{aligned}
& -F\left(t q_{1} a+\left(1-t q_{1}\right) b, s c+(1-s) d\right)-F\left(t a+(1-t) b, s q_{2} c+\left(1-s q_{2}\right) d\right) \\
& +F(t a+(1-t) b, s c+(1-s) d)] .
\end{aligned}
$$

To conclude the proof, we need to calculate the integrals in the right side of (3.2). By the definition of $q_{1} q_{2}$-integrals we obtain that

$$
\begin{aligned}
& \int_{0}^{\frac{b-x}{b-a}} \int_{0}^{\frac{d-y}{d-c}} \frac{b, d}{\partial_{q_{1}, q_{2}}^{2} F(t a+(1-t) b, s c+(1-s) d)} d_{q_{1}} t d_{q_{2}} s \\
& =\frac{1}{(b-a)(d-c)} \\
& \times\left[\sum _ { n = 0 } ^ { \infty } \sum _ { m = 0 } ^ { \infty } F \left(q_{1}^{n+1}\left(\frac{b-x}{b-a}\right) a+\left(1-q_{1}^{n+1}\left(\frac{b-x}{b-a}\right)\right) b,\right.\right. \\
& \left.q_{2}^{m+1}\left(\frac{d-y}{d-c}\right) c+\left(1-q_{2}^{m+1}\left(\frac{d-y}{d-c}\right)\right) d\right) \\
& -\sum_{n=0}^{\infty} \sum_{m=0}^{\infty} F\left(q_{1}^{n+1}\left(\frac{b-x}{b-a}\right) a+\left(1-q_{1}^{n+1}\left(\frac{b-x}{b-a}\right)\right) b,\right. \\
& \left.q_{2}^{m}\left(\frac{d-y}{d-c}\right) c+\left(1-q_{2}^{m}\left(\frac{d-y}{d-c}\right)\right) d\right) \\
& -\sum_{n=0}^{\infty} \sum_{m=0}^{\infty} F\left(q_{1}^{n}\left(\frac{b-x}{b-a}\right) a+\left(1-q_{1}^{n}\left(\frac{b-x}{b-a}\right)\right) b,\right. \\
& \left.q_{2}^{m+1}\left(\frac{d-y}{d-c}\right) c+\left(1-q_{2}^{m+1}\left(\frac{d-y}{d-c}\right)\right) d\right) \\
& +\sum_{n=0}^{\infty} \sum_{m=0}^{\infty} F\left(q_{1}^{n}\left(\frac{b-x}{b-a}\right) a+\left(1-q_{1}^{n}\left(\frac{b-x}{b-a}\right)\right) b,\right. \\
& \left.\left.q_{2}^{m}\left(\frac{d-y}{d-c}\right) c+\left(1-q_{2}^{m}\left(\frac{d-y}{d-c}\right)\right) d\right)\right] \\
& =\frac{1}{(b-a)(d-c)} \\
& \times\left[\sum _ { n = 0 } ^ { \infty } \left\{\sum _ { m = 0 } ^ { \infty } F \left(q_{1}^{n+1}\left(\frac{b-x}{b-a}\right) a+\left(1-q_{1}^{n+1}\left(\frac{b-x}{b-a}\right)\right) b,\right.\right.\right. \\
& \left.q_{2}^{m+1}\left(\frac{d-y}{d-c}\right) c+\left(1-q_{2}^{m+1}\left(\frac{d-y}{d-c}\right)\right) d\right) \\
& -\sum_{m=0}^{\infty} F\left(q_{1}^{n}\left(\frac{b-x}{b-a}\right) a+\left(1-q_{1}^{n}\left(\frac{b-x}{b-a}\right)\right) b,\right. \\
& \left.\left.q_{2}^{m+1}\left(\frac{d-y}{d-c}\right) c+\left(1-q_{2}^{m+1}\left(\frac{d-y}{d-c}\right)\right) d\right)\right\} \\
& +\sum_{n=0}^{\infty}\left\{\sum _ { m = 0 } ^ { \infty } F \left(q_{1}^{n}\left(\frac{b-x}{b-a}\right) a+\left(1-q_{1}^{n}\left(\frac{b-x}{b-a}\right)\right) b,\right.\right. \\
& \left.q_{2}^{m}\left(\frac{d-y}{d-c}\right) c+\left(1-q_{2}^{m}\left(\frac{d-y}{d-c}\right)\right) d\right)
\end{aligned}
$$




$$
\begin{aligned}
& -\sum_{m=0}^{\infty} F\left(q_{1}^{n+1}\left(\frac{b-x}{b-a}\right) a+\left(1-q_{1}^{n+1}\left(\frac{b-x}{b-a}\right)\right) b,\right. \\
& \left.\left.\left.q_{2}^{m}\left(\frac{d-y}{d-c}\right) c+\left(1-q_{2}^{m}\left(\frac{d-y}{d-c}\right)\right) d\right)\right\}\right] \\
= & \frac{1}{(b-a)(d-c)}\left[\sum_{n=0}^{\infty} F\left(q_{1}^{n+1}\left(\frac{b-x}{b-a}\right) a+\left(1-q_{1}^{n+1}\left(\frac{b-x}{b-a}\right)\right) b, d\right)\right. \\
& -\sum_{n=0}^{\infty} F\left(q_{1}^{n}\left(\frac{b-x}{b-a}\right) a+\left(1-q_{1}^{n}\left(\frac{b-x}{b-a}\right)\right) b, d\right) \\
& +\sum_{n=0}^{\infty} F\left(q_{1}^{n}\left(\frac{b-x}{b-a}\right) a+\left(1-q_{1}^{n}\left(\frac{b-x}{b-a}\right)\right) b, y\right) \\
& \left.-\sum_{n=0}^{\infty} F\left(q_{1}^{n+1}\left(\frac{b-x}{b-a}\right) a+\left(1-q_{1}^{n+1}\left(\frac{b-x}{b-a}\right)\right) b, y\right)\right] \\
= & \frac{1}{(b-a)(d-c)}[F(b, d)-F(x, d)-F(b, y)+F(x, y)] .
\end{aligned}
$$

Similarly, we have

$$
\begin{aligned}
& \int_{0}^{\frac{b-x}{b-a}} \int_{0}^{1} \frac{b, d}{\partial_{q_{1}, q_{2}}^{2} F(t a+(1-t) b, s c+(1-s) d)} d_{q_{1}} t d_{q_{2}} s \\
& =\frac{1}{(b-a)(d-c)}[F(b, d)-F(x, d)-F(b, c)+F(x, c)], \\
& \int_{0}^{1} \int_{0}^{\frac{d-y}{d-c}} \frac{b, d}{\partial_{q_{1}, q_{2}}^{2} F(t a+(1-t) b, s c+(1-s) d)} d_{q_{1}} t d_{q_{2}} s \\
& =\frac{1}{(b-a)(d-c)}[F(b, d)-F(b, y)-F(a, d)+F(a, y)], \\
& \int_{0}^{1} \int_{0}^{1} \frac{b, d}{\partial_{q_{1}, q_{2}}^{2} F(t a+(1-t) b, s c+(1-s) d)} d_{q_{1}} t d_{q_{2}} s \\
& =\frac{1}{(b-a)(d-c)}[F(b, d)-F(a, d)-F(b, c)+F(a, c)] \text {. }
\end{aligned}
$$

Additionally, we have

$$
\begin{aligned}
& \int_{0}^{\frac{b-x}{b-a}} \int_{0}^{1} s \frac{b, d}{{ }^{b} \partial_{q_{1}, q_{2}}^{2} F(t a+(1-t) b, s c+(1-s) d)} d_{q_{1}} t d_{q_{2}} s \\
& =\frac{1}{(b-a)(d-c)} \\
& \times\left[\sum_{n=0}^{\infty} \sum_{m=0}^{\infty} q_{2}^{m} F\left(q_{1}^{n+1}\left(\frac{b-x}{b-a}\right) a+\left(1-q_{1}^{n+1}\left(\frac{b-x}{b-a}\right)\right) b, q_{2}^{m+1} c+\left(1-q_{2}^{m+1}\right) d\right)\right. \\
& -\sum_{n=0}^{\infty} \sum_{m=0}^{\infty} q_{2}^{m} F\left(q_{1}^{n+1}\left(\frac{b-x}{b-a}\right) a+\left(1-q_{1}^{n+1}\left(\frac{b-x}{b-a}\right)\right) b, q_{2}^{m} c+\left(1-q_{2}^{m}\right) d\right)
\end{aligned}
$$


Ali et al. Advances in Difference Equations

(2021) 2021:25

Page 10 of 26

$$
\begin{aligned}
& -\sum_{n=0}^{\infty} \sum_{m=0}^{\infty} q_{2}^{m} F\left(q_{1}^{n}\left(\frac{b-x}{b-a}\right) a+\left(1-q_{1}^{n}\left(\frac{b-x}{b-a}\right)\right) b, q_{2}^{m+1} c+\left(1-q_{2}^{m+1}\right) d\right) \\
& \left.+\sum_{n=0}^{\infty} \sum_{m=0}^{\infty} q_{2}^{m} F\left(q_{1}^{n}\left(\frac{b-x}{b-a}\right) a+\left(1-q_{1}^{n}\left(\frac{b-x}{b-a}\right)\right) b, q_{2}^{m} c+\left(1-q_{2}^{m+1}\right) d\right)\right] \\
= & \frac{1}{(b-a)(d-c)} \\
& \times\left[\sum_{m=0}^{\infty} q_{2}^{m} F\left(b, q_{2}^{m+1} c+\left(1-q_{2}^{m+1}\right) d\right)-\sum_{m=0}^{\infty} q_{2}^{m} F\left(b, q_{2}^{m} c+\left(1-q_{2}^{m}\right) d\right)\right. \\
& \left.+\sum_{m=0}^{\infty} q_{2}^{m} F\left(x, q_{2}^{m} c+\left(1-q_{2}^{m}\right) d\right)-\sum_{m=0}^{\infty} q_{2}^{m} F\left(x, q_{2}^{m+1} c+\left(1-q_{2}^{m+1}\right) d\right)\right] \\
= & \left.\frac{1}{(b-a)(d-c)}\right] \\
& \times\left[\frac{1-q_{2}}{q_{2}} \sum_{m=0}^{\infty} q_{2}^{m} F\left(b, q_{2}^{m} c+\left(1-q_{2}^{m}\right) d\right)-\frac{1}{q_{2}} F(b, c)\right. \\
& \left.-\frac{1}{q_{2}} F(b, c)+\frac{1}{q_{2}} F(x, c)\right] . \\
& \left.-\frac{1-q_{2}}{q_{2}} \sum_{m=0}^{\infty} q_{2}^{m} F\left(x, q_{2}^{m} c+\left(1-q_{2}^{m}\right) d\right)+\frac{1}{q_{2}} F(x, c)\right] \\
(b-a)(d-c) & \frac{1}{q_{2}(d-c)} \int_{c}^{d} F(b, s)^{d} d_{q_{2}} s-\frac{1}{q_{2}(d-c)} \int_{c}^{d} F(x, s)^{d} d_{q_{2}} s
\end{aligned}
$$

By similar operations we have

$$
\begin{aligned}
& \int_{0}^{1} \int_{0}^{\frac{d-y}{d-c}} t \frac{b, d}{{ }^{b} \partial_{q_{1}, q_{2}}^{2} F(t a+(1-t) b, s c+(1-s) d)} d_{q_{1}} t d_{q_{2}} s \\
& =\frac{1}{(b-a)(d-c)}\left[\frac{1}{q_{1}(b-a)} \int_{a}^{b} F(t, d)^{b} d_{q_{1}} t-\frac{1}{q_{1}(b-a)} \int_{a}^{b} F(t, y)^{b} d_{q_{1}} t\right. \\
& \left.-\frac{1}{q_{1}} F(a, d)+\frac{1}{q_{2}} F(a, y)\right] \text {, } \\
& \int_{0}^{1} \int_{0}^{1} s \frac{b, d}{{ }^{b} \partial_{q_{1}, q_{2}}^{2} F(t a+(1-t) b, s c+(1-s) d)} d_{q_{1}} t d_{q_{2}} s \\
& =\frac{1}{(b-a)(d-c)}\left\{\frac{1}{q_{2}(d-c)} \int_{c}^{d} F(b, s)^{d} d_{q_{2}} s-\frac{1}{q_{2}(d-c)} \int_{c}^{d} F(a, s)^{d} d_{q_{2}} s\right. \\
& \left.-\frac{1}{q_{2}} F(b, c)+\frac{1}{q_{2}} F(a, c)\right\}, \\
& \int_{0}^{1} \int_{0}^{1} t \frac{b, d}{\partial_{q_{1}, q_{2}}^{2} F(t a+(1-t) b, s c+(1-s) d)} d_{q_{1}} t d_{q_{2}} s \\
& =\frac{1}{(b-a)(d-c)}\left\{\frac{1}{q_{1}(b-a)} \int_{a}^{b} F(t, d)^{b} d_{q_{1}} t-\frac{1}{q_{1}(b-a)} \int_{a}^{b} F(t, c)^{b} d_{q_{1}} t\right. \\
& \left.-\frac{1}{q_{1}} F(a, d)+\frac{1}{q_{1}} F(a, c)\right\} \text {, }
\end{aligned}
$$


Ali et al. Advances in Difference Equations

(2021) 2021:25

Page 11 of 26

and

$$
\begin{aligned}
& \int_{0}^{1} \int_{0}^{1} t s \frac{b, d}{\partial_{q_{1}, q_{2}}^{2} F(t a+(1-t) b, s c+(1-s) d)} d_{q_{1}} t d_{q_{2}} s \\
& =\frac{1}{(b-a)(d-c)}\left\{\sum_{m=0}^{\infty} \sum_{n=0}^{\infty} q_{1}^{n} q_{2}^{m} F\left(q_{1}^{n+1} a+\left(1-q_{1}^{n+1}\right) b, q_{2}^{m+1} c+\left(1-q_{2}^{m+1}\right) d\right)\right. \\
& -\sum_{m=0}^{\infty} \sum_{n=0}^{\infty} q_{1}^{n} q_{2}^{m} F\left(q_{1}^{n+1} a+\left(1-q_{1}^{n+1}\right) b, q_{2}^{m} c+\left(1-q_{2}^{m}\right) d\right) \\
& -\sum_{m=0}^{\infty} \sum_{n=0}^{\infty} q_{1}^{n} q_{2}^{m} F\left(q_{1}^{n} a+\left(1-q_{1}^{n}\right) b, q_{2}^{m+1} c+\left(1-q_{2}^{m+1}\right) d\right) \\
& \left.+\sum_{m=0}^{\infty} \sum_{n=0}^{\infty} q_{1}^{n} q_{2}^{m} F\left(q_{1}^{n} a+\left(1-q_{1}^{n}\right) b, q_{2}^{m} c+\left(1-q_{2}^{m}\right) d\right)\right\} \\
& =\frac{1}{(b-a)(d-c)}\left\{\frac { 1 } { q _ { 1 } q _ { 2 } } \left[\sum_{m=0}^{\infty} \sum_{n=0}^{\infty} q_{1}^{n} q_{2}^{m} F\left(q_{1}^{n} a+\left(1-q_{1}^{n}\right) b, q_{2}^{m} c+\left(1-q_{2}^{m}\right) d\right)\right.\right. \\
& \left.-\sum_{m=0}^{\infty} q_{2}^{m} F\left(a, q_{2}^{m} c+\left(1-q_{2}^{m}\right) d\right)-\sum_{n=0}^{\infty} q_{1}^{n} F\left(q_{1}^{n} a+\left(1-q_{1}^{n}\right) b, c\right)+F(a, c)\right] \\
& -\frac{1}{q_{1}}\left[\sum_{m=0}^{\infty} \sum_{n=0}^{\infty} q_{1}^{n} q_{2}^{m} F\left(q_{1}^{n} a+\left(1-q_{1}^{n}\right) b, q_{2}^{m} c+\left(1-q_{2}^{m}\right) d\right)\right. \\
& \left.-\sum_{m=0}^{\infty} q_{2}^{m} F\left(a, q_{2}^{m} c+\left(1-q_{2}^{m}\right) d\right)\right] \\
& -\frac{1}{q_{2}}\left[\sum_{m=0}^{\infty} \sum_{n=0}^{\infty} q_{1}^{n} q_{2}^{m} F\left(q_{1}^{n} a+\left(1-q_{1}^{n}\right) b, q_{2}^{m} c+\left(1-q_{2}^{m}\right) d\right)\right. \\
& \left.-\sum_{n=0}^{\infty} q_{1}^{n} F\left(q_{1}^{n} a+\left(1-q_{1}^{n}\right) b, c\right)\right] \\
& \left.+\sum_{m=0}^{\infty} \sum_{n=0}^{\infty} q_{1}^{n} q_{2}^{m} F\left(q_{1}^{n} a+\left(1-q_{1}^{n}\right) b, q_{2}^{m} c+\left(1-q_{2}^{m}\right) d\right)\right\} \\
& =\frac{1}{(b-a)(d-c)}\left\{\frac{\left(1-q_{1}\right)\left(1-q_{2}\right)}{q_{1} q_{2}} \sum_{m=0}^{\infty} \sum_{n=0}^{\infty} q_{1}^{n} q_{2}^{m} F\left(q_{1}^{n} a+\left(1-q_{1}^{n}\right) b, q_{2}^{m} c+\left(1-q_{2}^{m}\right) d\right)\right. \\
& -\frac{1-q_{2}}{q_{1} q_{2}} \sum_{m=0}^{\infty} q_{2}^{m} F\left(a, q_{2}^{m} c+\left(1-q_{2}^{m}\right) d\right) \\
& \left.-\frac{1-q_{1}}{q_{1} q_{2}} \sum_{n=0}^{\infty} q_{1}^{n} F\left(q_{1}^{n} a+\left(1-q_{1}^{n}\right) b, c\right)+\frac{1}{q_{1} q_{2}} F(a, c)\right\} \\
& =\frac{1}{(b-a)(d-c)}\left\{\frac{1}{q_{1} q_{2}(b-a)(d-c)} \int_{a}^{b} \int_{c}^{d} F(t, s)^{b} d_{q_{1}} t^{d} d_{q_{2}} s\right. \\
& \left.-\frac{1}{q_{1} q_{2}(b-a)} \int_{a}^{b} F(t, c)^{b} d_{q_{1}} t-\frac{1}{q_{1} q_{2}(d-c)} \int_{c}^{d} F(a, s)^{d} d_{q_{2}} s+\frac{1}{q_{1} q_{2}} F(a, c)\right\} .
\end{aligned}
$$


Now from (3.4)-(3.12) we obtain the following relations:

$$
\begin{aligned}
I_{1}= & \frac{1}{(b-a)(d-c)}[F(b, d)-F(x, d)-F(b, y)+F(x, y)], \\
I_{2}= & \frac{1}{(b-a)(d-c)} \\
& \times\left[\frac{1}{d-c} \int_{c}^{d} F(b, s)^{d} d_{q_{2}} s-\frac{1}{d-c} \int_{c}^{d} F(x, s)^{d} d_{q_{2}} s-F(b, d)+F(x, d)\right], \\
I_{3}= & \frac{1}{(b-a)(d-c)} \\
& \times\left[\frac{1}{b-a} \int_{a}^{b} F(t, d)^{b} d_{q_{1}} t-\frac{1}{b-a} \int_{a}^{b} F(t, y)^{b} d_{q_{1}} t-F(b, d)+F(b, y)\right], \\
I_{4}= & \frac{1}{(b-a)(d-c)}\left[\frac{1}{(b-a)(d-c)} \int_{a}^{b} \int_{c}^{d} F(t, s)^{b} d_{q_{1}} t^{d} d_{q_{2}} s-\frac{1}{b-a} \int_{a}^{b} F(t, d)^{b} d_{q_{1}} t\right. \\
& \left.-\frac{1}{d-c} \int_{c}^{d} F(b, s)^{d} d_{q_{2}} s+F(b, d)\right]^{b}
\end{aligned}
$$

which finishes the proof.

\section{Some new quantum Ostrowski-type integral inequalities}

In this section, we prove some new quantum Ostrowski-type inequalities for $q_{1} q_{2}$ differentiable coordinated convex functions using the lemma proved in the last section.

Theorem 5 Suppose that the assumptions of Lemma 3 hold. If $\left|\frac{b, \partial_{q_{1}, q_{2}}^{2} F(t, s)}{b_{q_{1}} t^{d} \partial_{q_{2}} s}\right|^{p_{1}}, p_{1}>1$, is coordinated convex on $[a, b] \times[c, d]$, then we have the inequality

$$
\begin{aligned}
& \mid \frac{1}{(b-a)(d-c)} \int_{a}^{b} \int_{c}^{d} F(t, s)^{b} d_{q_{1}} t^{d} d_{q_{2}} s-\frac{1}{b-a} \int_{a}^{b} F(t, y)^{b} d_{q_{1}} t \\
& -\frac{1}{d-c} \int_{c}^{d} F(x, s)^{d} d_{q_{2}} s+F(x, y) \\
& \leq(b-a)(d-c)\left[A_{1}^{1-\frac{1}{p_{1}}}\left(a, b, q_{1}, x\right) A_{1}^{1-\frac{1}{p_{1}}}\left(c, d, q_{2}, y\right)\right. \\
& \times\left\{A _ { 2 } ( a , b , q _ { 1 } , x ) \left(A_{2}\left(c, d, q_{2}, y\right)\left|\frac{{ }^{b, d} \partial_{q_{1}, q_{2}}^{2} F(a, c)}{{ }^{b} \partial_{q_{1}} t^{d} \partial_{q_{2}} s}\right|^{p_{1}}\right.\right. \\
& \left.+A_{3}\left(c, d, q_{2}, y\right)\left|\frac{b, d}{b \partial_{q_{1}, q_{2}}^{2} t^{d} \partial_{q_{2}} s}\right|^{p_{1}}\right) \\
& +A_{3}\left(a, b, q_{1}, x\right)\left(A_{2}\left(c, d, q_{2}, y\right)\left|\frac{{ }^{b, d} \partial_{q_{1}, q_{2}}^{2} F(b, c)}{{ }^{b} \partial_{q_{1}} t^{d} \partial_{q_{2}} s}\right|^{p_{1}}\right. \\
& \left.\left.+A_{3}\left(c, d, q_{2}, y\right)\left|\frac{b, d}{\partial_{q_{1}, q_{2}}^{2} F(b, d)}\right|^{b_{q_{1}} t^{d} \partial_{q_{2}} s}\right)\right\}^{\frac{1}{p_{1}}} \\
& +A_{1}^{1-\frac{1}{p_{1}}}\left(a, b, q_{1}, x\right) A_{4}^{1-\frac{1}{p_{1}}}\left(c, d, q_{2}, y\right)
\end{aligned}
$$


Ali et al. Advances in Difference Equations

(2021) 2021:25

Page 13 of 26

$$
\begin{aligned}
& \times\left\{A _ { 2 } ( a , b , q _ { 1 } , x ) \left(A_{5}\left(c, d, q_{2}, y\right) \mid \frac{b, d}{\partial_{q_{1}, q_{2}}^{2} F(a, c)} \frac{{ }^{b} \partial_{q_{1}} t^{d} \partial_{q_{2}} s}{p_{1}}\right.\right. \\
& \left.+A_{6}\left(c, d, q_{2}, y\right)\left|\frac{{ }^{b, d} \partial_{q_{1}, q_{2}}^{2} F(a, d)}{{ }^{b} \partial_{q_{1}} t^{d} \partial_{q_{2}} s}\right|^{p_{1}}\right) \\
& +A_{3}\left(a, b, q_{1}, x\right)\left(\left.A_{5}\left(c, d, q_{2}, y\right)\left|\frac{b, d}{\partial_{q_{1}, q_{2}}^{2} F(b, c)}\right|^{b \partial_{q_{1}} t^{d} \partial_{q_{2}} s}\right|^{p_{1}}\right. \\
& \left.\left.+A_{6}\left(c, d, q_{2}, y\right)\left|\frac{b, d}{\partial_{q_{1}, q_{2}}^{2} F(b, d)}\right|^{{ }^{b} \partial_{q_{1}} t^{d} \partial_{q_{2}} s}\right)\right\}^{\frac{1}{p_{1}}} \\
& +A_{4}^{1-\frac{1}{p_{1}}}\left(a, b, q_{1}, x\right) A_{1}^{1-\frac{1}{p_{1}}}\left(c, d, q_{2}, y\right)
\end{aligned}
$$

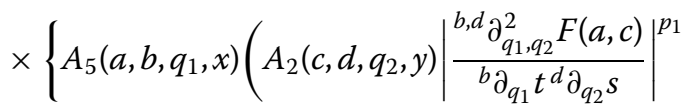

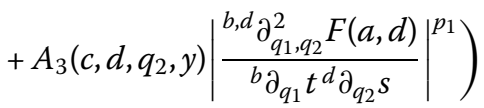

$$
\begin{aligned}
& +A_{6}\left(a, b, q_{1}, x\right)\left(\left.A_{2}\left(c, d, q_{2}, y\right)\left|\frac{b, d}{\frac{b}{\partial_{q_{1}, q_{2}}^{2} F(b, c)}}\right|^{b \partial_{q_{1}} t^{d} \partial_{q_{2}} s}\right|^{p_{1}}\right. \\
& \left.\left.+A_{3}\left(c, d, q_{2}, y\right)\left|\frac{b, d}{{ }^{b} \partial_{q_{1}, q_{2}}^{2} F(b, d)}\right|^{b_{q_{1}} t^{d} \partial_{q_{2}} s}\right)\right\}^{\frac{1}{p_{1}}} \\
& +A_{4}^{1-\frac{1}{p_{1}}}\left(a, b, q_{1}, x\right) A_{4}^{1-\frac{1}{p_{1}}}\left(c, d, q_{2}, y\right) \\
& \times\left\{A _ { 5 } ( a , b , q _ { 1 } , x ) \left(A_{5}\left(c, d, q_{2}, y\right) \mid \frac{b, d}{\partial_{q_{1}, q_{2}}^{2} F(a, c)} \frac{{ }^{b} \partial_{q_{1}} t^{d} \partial_{q_{2}} s}{p_{1}}\right.\right. \\
& \left.+A_{6}\left(c, d, q_{2}, y\right)\left|\frac{b_{q_{1}, q_{2}} F(a, d)}{{ }^{b} \partial_{q_{1}} t^{d} \partial_{q_{2}} s}\right|^{p_{1}}\right) \\
& +A_{6}\left(a, b, q_{1}, x\right)\left(\left.A_{5}\left(c, d, q_{2}, y\right)\left|\frac{b, d}{\partial_{q_{1}, q_{2}}^{2} F(b, c)}\right|^{b \partial_{q_{1}} t^{d} \partial_{q_{2}} s}\right|^{p_{1}}\right. \\
& \left.\left.\left.+A_{6}\left(c, d, q_{2}, y\right)\left|\frac{b, d}{\partial_{q_{1}, q_{2}}^{2} F(b, d)}\right|^{b_{q_{1}} t^{d} \partial_{q_{2}} s}\right)\right\}^{\frac{1}{p_{1}}}\right] \text {, }
\end{aligned}
$$

where

$$
\begin{aligned}
A_{1}(u, v, q, z) & =\int_{0}^{\frac{v-z}{v-u}} q t d_{q} t=\frac{q}{1+q}\left(\frac{v-z}{v-u}\right)^{2}, \\
A_{2}(u, v, q, z) & =\int_{0}^{\frac{v-z}{v-u}} q t^{2} d_{q} t=\frac{q}{1+q+q^{2}}\left(\frac{v-z}{v-u}\right)^{3}, \\
A_{3}(u, v, q, z) & =\int_{0}^{\frac{v-z}{v-u}} q t d_{q} t-\int_{0}^{\frac{v-z}{v-u}} q t^{2} d_{q} t=A_{1}(u, v, q, z)-A_{2}(u, v, q, z), \\
A_{4}(u, v, q, z) & =\int_{\frac{v-z}{v-u}}^{1}(1-q t) d_{q} t=\int_{0}^{1}(1-q t) d_{q} t-\int_{0}^{\frac{v-z}{v-u}}(1-q t) d_{q} t \\
& =\frac{1-q}{1+q}\left(\frac{z-u}{v-u}\right)+\frac{q}{1+q}\left(\frac{z-u}{v-u}\right)^{2},
\end{aligned}
$$


Ali et al. Advances in Difference Equations

(2021) 2021:25

Page 14 of 26

$$
\begin{aligned}
A_{5}(u, v, q, z)= & \int_{\frac{v-z}{v-u}}^{1}\left(t-q t^{2}\right) d_{q} t=\int_{0}^{1}\left(t-q t^{2}\right) d_{q} t-\int_{0}^{\frac{v-z}{v-u}}\left(t-q t^{2}\right) d_{q} t \\
= & \frac{1}{(1+q)\left(1+q+q^{2}\right)}-\frac{1}{1+q}\left(\frac{v-z}{v-u}\right)^{2}+\frac{q}{1+q+q^{2}}\left(\frac{v-z}{v-u}\right)^{3}, \\
A_{6}(u, v, q, z)= & \int_{\frac{v-z}{v-u}}^{1}(1-t)(1-q t) d_{q} t \\
= & \int_{0}^{1}(1-t)(1-q t) d_{q} t-\int_{0}^{\frac{v-z}{v-u}}(1-t)(1-q t) d_{q} t \\
= & \int_{0}^{1}(1-q t) d_{q} t-\int_{0}^{1}\left(t-q t^{2}\right) d_{q} t \\
& -\int_{0}^{\frac{v-z}{v-u}}(1-q t) d_{q} t+\int_{0}^{\frac{v-z}{v-u}}\left(t-q t^{2}\right) d_{q} t \\
= & A_{4}(u, v, q, z)-A_{5}(u, v, q, z),
\end{aligned}
$$

and $q_{1}, q_{2} \in(0,1)$.

Proof By taking the modulus in Lemma 3 we have

$$
\begin{aligned}
& \mid \frac{1}{(b-a)(d-c)} \int_{a}^{b} \int_{c}^{d} F(t, s)^{b} d_{q_{1}} t^{d} d_{q_{2}} s-\frac{1}{b-a} \int_{a}^{b} F(t, y)^{b} d_{q_{1}} t \\
& -\frac{1}{d-c} \int_{c}^{d} F(x, s)^{d} d_{q_{2}} s+F(x, y) \\
& \leq(b-a)(d-c) \\
& \times \int_{0}^{1} \int_{0}^{1}\left|\Psi_{q_{1}}(t) \Psi_{q_{2}}(s)\right|\left|\frac{b_{q_{1}, q_{2}}^{b, d} F(t a+(1-t) b, s c+(1-s) d)}{b \partial_{q_{1}} t^{d} \partial_{q_{2}} s}\right| d_{q_{1}} t d_{q_{2}} s \\
& =(b-a)(d-c)
\end{aligned}
$$

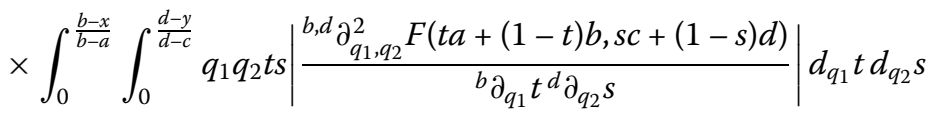

$$
\begin{aligned}
& +(b-a)(d-c)
\end{aligned}
$$

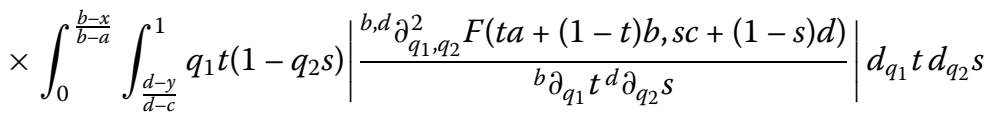

$$
\begin{aligned}
& +(b-a)(d-c)
\end{aligned}
$$

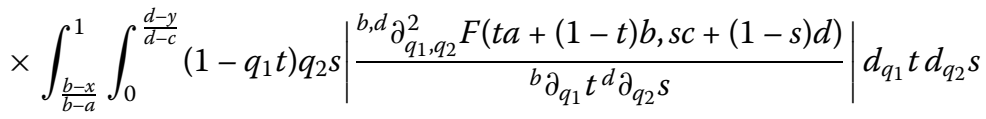

$$
\begin{aligned}
& +(b-a)(d-c) \\
& \times \int_{\frac{b-x}{b-a}}^{1} \int_{\frac{d-y}{d-c}}^{1}\left(1-q_{1} t\right)\left(1-q_{2} s\right)\left|\frac{\partial_{q_{1}, q_{2}}^{2} F(t a+(1-t) b, s c+(1-s) d)}{{ }^{b} \partial_{q_{1}} t^{d} \partial_{q_{2}} s}\right| d_{q_{1}} t d_{q_{2}} s .
\end{aligned}
$$


Ali et al. Advances in Difference Equations

(2021) $2021: 25$

Page 15 of 26

Applying the power mean inequality for quantum integrals, we obtain

$$
\begin{aligned}
& \mid \frac{1}{(b-a)(d-c)} \int_{a}^{b} \int_{c}^{d} F(t, s)^{b} d_{q_{1}} t^{d} d_{q_{2}} s-\frac{1}{b-a} \int_{a}^{b} F(t, y)^{b} d_{q_{1}} t \\
& -\frac{1}{d-c} \int_{c}^{d} F(x, s)^{d} d_{q_{2}} s+F(x, y) \\
& \leq(b-a)(d-c)\left(\int_{0}^{\frac{b-x}{b-a}} \int_{0}^{\frac{d-y}{d-c}} q_{1} q_{2} t s d_{q_{1}} t d_{q_{2}} s\right)^{1-\frac{1}{p_{1}}} \\
& \times\left(\int_{0}^{\frac{b-x}{b-a}} \int_{0}^{\frac{d-y}{d-c}} q_{1} q_{2} t s\left|\frac{b, d}{\partial_{q_{1}, q_{2}}^{2} F(t a+(1-t) b, s c+(1-s) d)}\right|^{{ }^{b} \partial_{q_{1}} t d \partial_{q_{2}} s} d_{q_{1}} t d_{q_{2}} s\right)^{\frac{1}{p_{1}}} \\
& +(b-a)(d-c)\left(\int_{0}^{\frac{b-x}{b-a}} \int_{\frac{d-y}{d-c}}^{1} q_{1} t\left(1-q_{2} s\right) d_{q_{1}} t d_{q_{2}} s\right)^{1-\frac{1}{p_{1}}} \\
& \times\left(\int_{0}^{\frac{b-x}{b-a}} \int_{\frac{d-y}{d-c}}^{1} q_{1} t\left(1-q_{2} s\right)\left|\frac{b, d}{\partial_{q_{1}, q_{2}}^{2} F(t a+(1-t) b, s c+(1-s) d)}\right|^{b_{1}} d_{q_{1}} t d_{q_{2}} s\right)^{\frac{1}{p_{1}}} \\
& +(b-a)(d-c)\left(\int_{\frac{b-x}{b-a}}^{1} \int_{0}^{\frac{d-y}{d-c}}\left(1-q_{1} t\right) q_{2} s d_{q_{1}} t d_{q_{2}} s\right)^{1-\frac{1}{p_{1}}}
\end{aligned}
$$

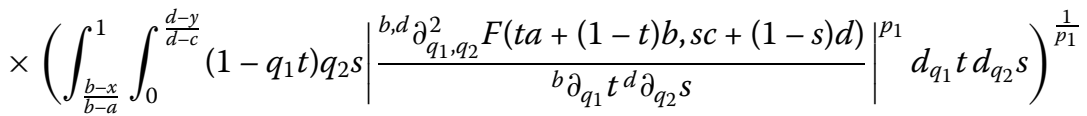

$$
\begin{aligned}
& +(b-a)(d-c)\left(\int_{\frac{b-x}{b-a}}^{1} \int_{\frac{d-y}{d-c}}^{1}\left(1-q_{1} t\right)\left(1-q_{2} s\right) d_{q_{1}} t d_{q_{2}} s\right)^{1-\frac{1}{p_{1}}} \\
& \times\left(\int_{\frac{b-x}{b-a}}^{1} \int_{\frac{d-y}{d-c}}^{1}\left(1-q_{1} t\right)\left(1-q_{2} s\right)\right.
\end{aligned}
$$

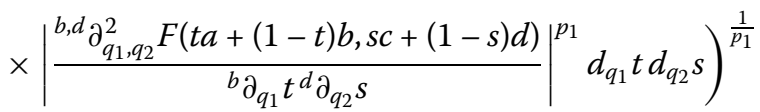

Now using the convexity of $\left|\frac{b, d \partial_{q_{1}, q_{2}}^{2} F(t, s)}{b_{q_{1}} t^{d} \partial_{q_{2}} s}\right|^{p_{1}}$, we obtain

$$
\begin{aligned}
& {\left[\int_{0}^{\frac{b-x}{b-a}} \int_{0}^{\frac{d-y}{d-c}} q_{1} q_{2} t s\left|\frac{b, d}{b \partial_{q_{1}, q_{2}}^{2} F(t a+(1-t) b, s c+(1-s) d)}\right|^{b_{q_{1}} t^{d} \partial_{q_{2}} s} d_{q_{1}} t d_{q_{2}} s\right]^{\frac{1}{p_{1}}}} \\
& \leq\left[\int _ { 0 } ^ { \frac { d - y } { d - c } } q _ { 2 } s \left\{\int _ { 0 } ^ { \frac { b - x } { b - a } } q _ { 1 } t \left(t\left|\frac{b, d}{\partial_{q_{1}, q_{2}}^{2} F(a, s c+(1-s) d)}\right|^{{ }^{p_{1}} \partial_{q_{1}} t d \partial_{q_{2}} s}\right.\right.\right.
\end{aligned}
$$

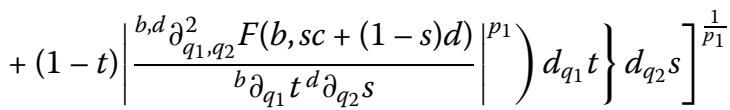

$$
\begin{aligned}
& =\left[\int _ { 0 } ^ { \frac { d - y } { d - c } } q _ { 2 } s \left\{A_{2}\left(a, b, q_{1}, x\right)\left|\frac{b_{q_{1}, q_{2}} F(a, s c+(1-s) d)}{{ }^{b} \partial_{q_{1}} t d \partial_{q_{2}} s}\right|^{p_{1}}\right.\right. \\
& \left.\left.+A_{3}\left(a, b, q_{1}, x\right)\left|\frac{b, d}{\partial_{q_{1}, q_{2}}^{2} F(b, s c+(1-s) d)}\right|^{{ }^{p_{1}} \partial_{q_{1}} t^{d} \partial_{q_{2}} s}\right\} d_{q_{2}} s\right]^{\frac{1}{p_{1}}}
\end{aligned}
$$


Ali et al. Advances in Difference Equations

(2021) 2021:25

Page 16 of 26

$$
\begin{aligned}
& \leq\left[A_{2}\left(a, b, q_{1}, x\right) \int_{0}^{\frac{d-y}{d-c}} q_{2} s\left\{s\left|\frac{\partial_{q_{1}, q_{2}} F(a, c)}{b \partial_{q_{1}} t^{d} \partial_{q_{2}} s}\right|^{p_{1}}+(1-s)\left|\frac{{ }^{b, d} \partial_{q_{1}, q_{2}}^{2} F(a, d)}{b \partial_{q_{1}} t^{d} \partial_{q_{2}} s}\right|^{p_{1}}\right\}\right. \\
& \left.+A_{3}\left(a, b, q_{1}, x\right) \int_{0}^{\frac{d-y}{d-c}} q_{2} s\left\{s\left|\frac{b, d}{{ }^{2} \partial_{q_{1}, q_{2}}^{2} F(b, c)}\right|^{b_{q_{1}} t^{d} \partial_{q_{2}} s}+(1-s)\left|\frac{p_{1}}{b \partial_{q_{1}} t^{d} \partial_{q_{2}} s}\right|^{2}\right\}\right]^{\frac{1}{p_{1}}}
\end{aligned}
$$

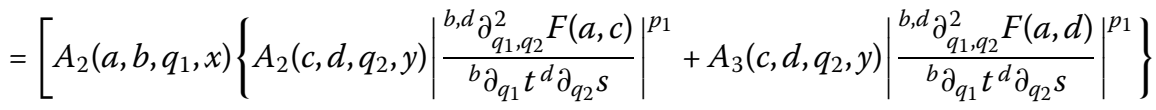

$$
\begin{aligned}
& +A_{3}\left(a, b, q_{1}, x\right)\left\{\left.A_{2}\left(c, d, q_{2}, y\right)\left|\frac{b, d}{\partial_{q_{1}, q_{2}}^{2} F(b, c)}\right|^{b_{q_{1}} t^{d} \partial_{q_{2}} s}\right|^{p_{1}}\right. \\
& +A_{3}\left(c, d, q_{2}, y\right)\left|\frac{b, d}{\partial_{q_{1}, q_{2}}^{2} F(b, d)} \frac{p_{1}}{b \partial_{q_{1}} t^{d} \partial_{q_{2}} s}\right|^{\frac{1}{p_{1}}} \text {. }
\end{aligned}
$$

By using similar operations we find

$$
\begin{aligned}
& {\left[\int_{0}^{\frac{b-x}{b-a}} \int_{\frac{d-y}{d-c}}^{1} q_{1} t\left(1-q_{2} s\right)\left|\frac{b, d}{\partial_{q_{1}, q_{2}}^{2} F(t a+(1-t) b, s c+(1-s) d)}\right|^{p_{1}} d_{q_{1}} t d_{q_{2}} s\right]^{\frac{1}{p_{1}}}} \\
& \leq\left[A _ { 2 } ( a , b , q _ { 1 } , x ) \left\{A_{5}\left(c, d, q_{2}, y\right)\left|\frac{b, d}{\partial_{q_{1}, q_{2}}^{2} F(a, c)}\right|^{b_{1} \partial_{q_{1}} t^{d} \partial_{q_{2}} s}\right.\right. \\
& \left.+A_{6}\left(c, d, q_{2}, y\right)\left|\frac{b, d}{\partial_{q_{1}, q_{2}}^{2} F(a, d)}\right|^{{ }^{p_{1}} \partial_{q_{1}} t^{d} \partial_{q_{2}} s}\right\} \\
& +A_{3}\left(a, b, q_{1}, x\right)\left\{A_{5}\left(c, d, q_{2}, y\right)\left|\frac{{ }^{b, d} \partial_{q_{1}, q_{2}}^{2} F(b, c)}{b \partial_{q_{1}} t^{d} \partial_{q_{2}} s}\right|^{p_{1}}\right.
\end{aligned}
$$

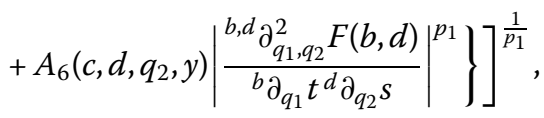

$$
\begin{aligned}
& {\left[\int_{\frac{b-x}{b-a}}^{1} \int_{0}^{\frac{d-y}{d-c}}\left(1-q_{1} t\right) q_{2} s\left|\frac{b, d}{\partial_{q_{1}, q_{2}}^{2} F(t a+(1-t) b, s c+(1-s) d)}\right|^{p_{1}} d_{q_{1}} t d_{q_{2}} s\right]^{\frac{1}{p_{1}}}} \\
& \leq\left[A _ { 5 } ( a , b , q _ { 1 } , x ) \left\{A_{2}\left(c, d, q_{2}, y\right)\left|\frac{b, d}{\partial_{q_{1}, q_{2}}^{2} F(a, c)}\right|^{b_{1}}\right.\right. \\
& \left.+A_{3}\left(c, d, q_{2}, y\right)\left|\frac{b, d}{\partial_{q_{1}, q_{2}}^{2} F(a, d)} \frac{p^{p_{1}}}{{ }^{2} \partial_{q_{1}} t^{d} \partial_{q_{2}} s}\right|^{3}\right\} \\
& +A_{6}\left(a, b, q_{1}, x\right)\left\{\left.A_{2}\left(c, d, q_{2}, y\right)\left|\frac{b, d}{\partial_{q_{1}, q_{2}}^{2} F(b, c)}\right|^{b \partial_{q_{1}} t^{d} \partial_{q_{2}} s}\right|^{p_{1}}\right.
\end{aligned}
$$

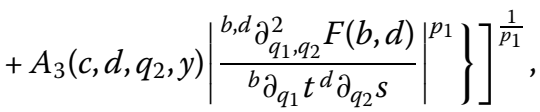

and

$$
\begin{aligned}
& {\left[\int_{\frac{b-x}{b-a}}^{1} \int_{\frac{d-y}{d-c}}^{1}\left(1-q_{1} t\right)\left(1-q_{2} s\right)\left|\frac{\partial_{q_{1}, q_{2}}^{2} F(t a+(1-t) b, s c+(1-s) d)}{{ }^{b} \partial_{q_{1}} t^{d} \partial_{q_{2}} s}\right|^{p_{1}} d_{q_{1}} t d_{q_{2}} s\right]^{\frac{1}{p_{1}}}} \\
& \quad \leq\left[A _ { 5 } ( a , b , q _ { 1 } , x ) \left\{A_{5}\left(c, d, q_{2}, y\right)\left|\frac{b^{b, d} \partial_{q_{1}, q_{2}}^{2} F(a, c)}{{ }^{b} \partial_{q_{1}} t^{d} \partial_{q_{2}} s}\right|^{p_{1}}\right.\right.
\end{aligned}
$$




$$
\begin{aligned}
& \left.+A_{6}\left(c, d, q_{2}, y\right)\left|\frac{b, d}{b \partial_{q_{1}, q_{2}}^{2} F(a, d)}\right|^{p_{q_{1}} t^{d} \partial_{q_{2}} s}\right\} \\
& +A_{6}\left(a, b, q_{1}, x\right)\left\{A_{5}\left(c, d, q_{2}, y\right)\left|\frac{{ }_{q_{1}, q_{2}} F(b, c)}{b \partial_{q_{1}} t^{d} \partial_{q_{2}} s}\right|^{p_{1}}\right. \\
& \left.\left.+A_{6}\left(c, d, q_{2}, y\right)\left|\frac{{ }^{b, d} \partial_{q_{1}, q_{2}}^{2} F(b, d)}{b \partial_{q_{1}} t^{d} \partial_{q_{2}} s}\right|^{p_{1}}\right\}\right]^{\frac{1}{p_{1}}} .
\end{aligned}
$$

We also observe that

$$
\begin{aligned}
& \left(\int_{0}^{\frac{b-x}{b-a}} \int_{0}^{\frac{d-y}{d-c}} q_{1} q_{2} t s d_{q_{1}} t d_{q_{2}} s\right)^{1-\frac{1}{p_{1}}}=\left(\left(\int_{0}^{\frac{b-x}{b-a}} q_{1} t d_{q_{1}} t\right)\left(\int_{0}^{\frac{d-y}{d-c}} q_{2} s d_{q_{2}} s\right)\right)^{1-\frac{1}{p_{1}}} \\
& =A_{1}^{1-\frac{1}{p_{1}}}\left(a, b, q_{1}, x\right) A_{1}^{1-\frac{1}{p_{1}}}\left(c, d, q_{2}, y\right), \\
& \left(\int_{0}^{\frac{b-x}{b-a}} \int_{\frac{d-y}{d-c}}^{1} q_{1} t\left(1-q_{2} s\right) d_{q_{1}} t d_{q_{2}} s\right)^{1-\frac{1}{p_{1}}}=A_{1}^{1-\frac{1}{p_{1}}}\left(a, b, q_{1}, x\right) A_{4}^{1-\frac{1}{p_{1}}}\left(c, d, q_{2}, y\right), \\
& \left(\int_{\frac{b-x}{b-a}}^{1} \int_{0}^{\frac{d-y}{d-c}}\left(1-q_{1} t\right) q_{2} s d_{q_{1}} t d_{q_{2}} s\right)^{1-\frac{1}{p_{1}}}=A_{4}^{1-\frac{1}{p_{1}}}\left(a, b, q_{1}, x\right) A_{1}^{1-\frac{1}{p_{1}}}\left(c, d, q_{2}, y\right), \\
& \left(\int_{\frac{b-x}{b-a}}^{1} \int_{\frac{d-y}{d-c}}^{1}\left(1-q_{1} t\right)\left(1-q_{2} s\right) d_{q_{1}} t d_{q_{2}} s\right)^{1-\frac{1}{p_{1}}}=A_{4}^{1-\frac{1}{p_{1}}}\left(a, b, q_{1}, x\right) A_{4}^{1-\frac{1}{p_{1}}}\left(c, d, q_{2}, y\right) .
\end{aligned}
$$

By (4.3)-(4.10) we obtain the desired inequality, which finishes the proof.

Theorem 6 Suppose that the assumptions of Lemma 3 hold. If $\left|\frac{b, d \partial_{q_{1}, q_{2}}^{2} F(t, s)}{b_{\partial_{1}} t^{d} \partial_{q_{2}} s}\right|^{p_{1}}$ is coordinated convex on $[a, b] \times[c, d]$, then we have the inequality

$$
\begin{aligned}
& \mid \frac{1}{(b-a)(d-c)} \int_{a}^{b} \int_{c}^{d} F(t, s)^{b} d_{q_{1}} t^{d} d_{q_{2}} s-\frac{1}{b-a} \int_{a}^{b} F(t, y)^{b} d_{q_{1}} t \\
& -\frac{1}{d-c} \int_{c}^{d} F(x, s)^{d} d_{q_{2}} s+F(x, y) \\
& \leq(b-a)(d-c) \\
& \times\left[\left(\frac{b-x}{b-a}\right)^{1+\frac{1}{r_{1}}}\left(\frac{d-y}{d-c}\right)^{1+\frac{1}{r_{1}}}\left(\frac{q_{1}}{\left[r_{1}+1\right]_{q_{1}}}\right)^{\frac{1}{r_{1}}}\left(\frac{q_{2}}{\left[r_{1}+1\right]_{q_{2}}}\right)^{\frac{1}{r_{1}}}\right) \\
& \times\left\{\left|\frac{b, d}{{ }^{b} \partial_{q_{1}} t^{d} \partial_{q_{2}} s}\right|^{p_{1}} \frac{1}{[2]_{q_{1}}[2]_{q_{2}}}\left(\frac{b-x}{b-a}\right)^{2}\left(\frac{d-y}{d-c}\right)^{2}\right. \\
& +\left|\frac{b, d}{{ }^{b} \partial_{q_{1}} t^{d} \partial_{q_{2}} s}\right|^{2} \frac{1}{[2]_{q_{1}}}\left(\frac{b-x}{b-a}\right)^{2}\left(\frac{d-y}{d-c}-\frac{1}{[2]_{q_{2}}}\left(\frac{d-y}{d-c}\right)^{2}\right) \\
& +\left|\frac{b, d}{{ }^{b} \partial_{q_{1}} t^{d} \partial_{q_{2}} s}\right|^{2} \frac{1 b, c)}{[2]_{q_{2}}}\left(\frac{d-y}{d-c}\right)^{2}\left(\frac{b-x}{b-a}-\frac{1}{[2]_{q_{1}}}\left(\frac{b-x}{b-a}\right)^{2}\right) \\
& +\left|\frac{b, d}{{ }^{b} \partial_{q_{1}, q_{2}}^{2} t^{d} \partial_{q_{2}} s}\right|^{p_{1}}\left(\frac{b-x}{b-a}-\frac{1}{[2]_{q_{1}}}\left(\frac{b-x}{b-a}\right)^{2}\right) \\
& \left.\times\left(\frac{d-y}{d-c}-\frac{1}{[2]_{q_{2}}}\left(\frac{d-y}{d-c}\right)^{2}\right)\right\}^{\frac{1}{p_{1}}}
\end{aligned}
$$


Ali et al. Advances in Difference Equations

(2021) $2021: 25$

Page 18 of 26

$$
\begin{aligned}
& +\left(\left(\frac{d-y}{d-c}\right)^{1+\frac{1}{r_{1}}}\left(\frac{q_{2}}{\left[r_{1}+1\right]_{q_{2}}}\right)^{\frac{1}{r_{1}}}\left(\int_{\frac{b-x}{b-a}}^{1}\left(1-q_{1} t\right)^{r_{1}} d_{q_{1}} t\right)^{\frac{1}{r_{1}}}\right)
\end{aligned}
$$

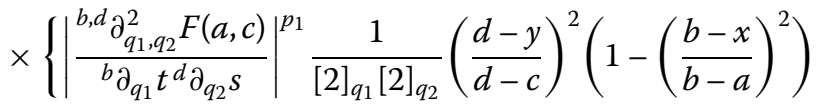

$$
\begin{aligned}
& +\left|\frac{b, d}{{ }^{b} \partial_{q_{1}, q_{2}}^{2} F(a, d)}\right|^{d} \partial_{q_{2}} s \quad \frac{1}{[2]_{q_{1}}}\left(1-\left(\frac{b-x}{b-a}\right)^{2}\right)\left(\frac{d-y}{d-c}-\frac{1}{[2]_{q_{2}}}\left(\frac{d-y}{d-c}\right)^{2}\right)
\end{aligned}
$$

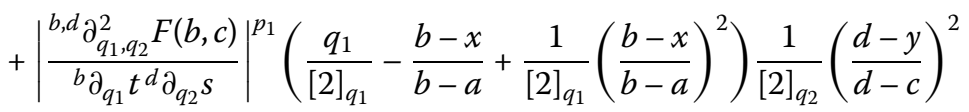

$$
\begin{aligned}
& +\left|\frac{\partial_{q_{1}, q_{2}}^{2} F(b, d)}{{ }^{b} \partial_{q_{1}} t^{d} \partial_{q_{2}} s}\right|^{p_{1}}\left(\frac{q_{1}}{[2]_{q_{1}}}-\frac{b-x}{b-a}+\frac{1}{[2]_{q_{1}}}\left(\frac{b-x}{b-a}\right)^{2}\right) \\
& \left.\times\left(\frac{d-y}{d-c}-\frac{1}{[2]_{q_{2}}}\left(\frac{d-y}{d-c}\right)^{2}\right)\right\}^{\frac{1}{p_{1}}} \\
& +\left(\left(\frac{b-x}{b-a}\right)^{1+\frac{1}{r_{1}}}\left(\frac{q_{1}}{\left[r_{1}+1\right]_{q_{1}}}\right)^{\frac{1}{r_{1}}}\left(\int_{\frac{d-y}{d-c}}^{1}\left(1-q_{2} s\right)^{r_{1}} d_{q_{2}} s\right)^{\frac{1}{r_{1}}}\right)
\end{aligned}
$$

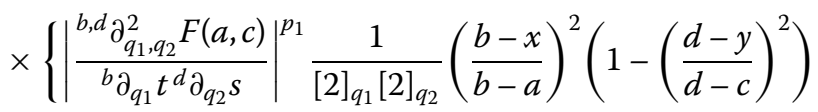

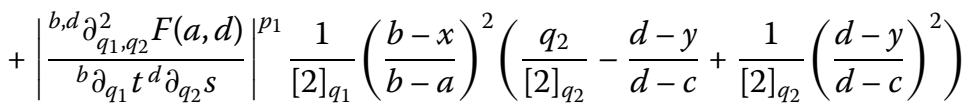

$$
\begin{aligned}
& +\left|\frac{b, d}{{ }^{b} \partial_{q_{1}, q_{2}}^{2} t^{d} \partial_{q_{2}} s}\right|^{p_{1}} \frac{1}{[2]_{q_{2}}}\left(1-\left(\frac{d-y}{d-c}\right)^{2}\right)\left(\frac{b-x}{b-a}-\frac{1}{[2]_{q_{1}}}\left(\frac{b-x}{b-a}\right)^{2}\right)
\end{aligned}
$$

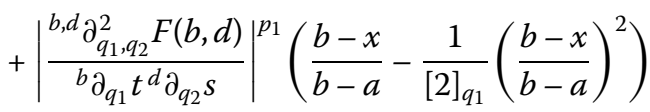

$$
\begin{aligned}
& \left.\times\left(\frac{q_{2}}{[2]_{q_{2}}}-\frac{d-y}{d-c}+\frac{1}{[2]_{q_{2}}}\left(\frac{d-y}{d-c}\right)^{2}\right)\right\}^{\frac{1}{p_{1}}} \\
& +\left(\left(\int_{\frac{b-x}{b-a}}^{1}\left(1-q_{1} t\right)^{r_{1}} d_{q_{1}} t\right)^{\frac{1}{r_{1}}}\left(\int_{\frac{d-y}{d-c}}^{1}\left(1-q_{2} s\right)^{r_{1}} d_{q_{2}} s\right)^{\frac{1}{r_{1}}}\right)
\end{aligned}
$$

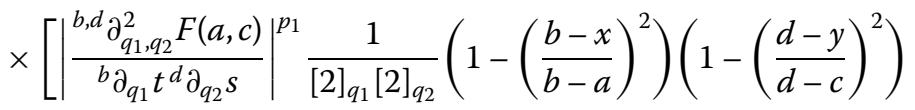

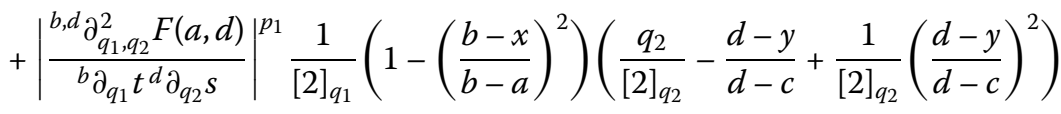

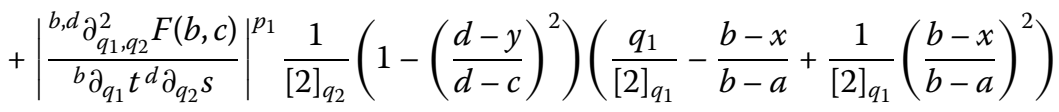

$$
\begin{aligned}
& +\left|\frac{{ }^{b, d} \partial_{q_{1}, q_{2}}^{2} F(b, d)}{b \partial_{q_{1}} t^{d} \partial_{q_{2}} s}\right|^{p_{1}}\left(\frac{q_{1}}{[2]_{q_{1}}}-\frac{b-x}{b-a}+\frac{1}{[2]_{q_{1}}}\left(\frac{b-x}{b-a}\right)^{2}\right) \\
& \left.\left.\times\left(\frac{q_{2}}{[2]_{q_{2}}}-\frac{d-y}{d-c}+\frac{1}{[2]_{q_{2}}}\left(\frac{d-y}{d-c}\right)^{2}\right)\right]^{\frac{1}{p_{1}}}\right] \text {, }
\end{aligned}
$$

where $q_{1}, q_{2} \in(0,1)$ and $\frac{1}{r_{1}}+\frac{1}{p_{1}}=1, p_{1}>1$. 
Proof Applying the well-known Hölder inequality for $q_{1} q_{2}$-integrals to the integrals in the right side of (4.2), we find

$$
\begin{aligned}
& \mid \frac{1}{(b-a)(d-c)} \int_{a}^{b} \int_{c}^{d} F(t, s)^{b} d_{q_{1}} t^{d} d_{q_{2}} s-\frac{1}{b-a} \int_{a}^{b} F(t, y)^{b} d_{q_{1}} x \\
& -\frac{1}{d-c} \int_{c}^{d} F(x, s)^{d} d_{q_{2}} s+F(x, y) \\
& \leq(b-a)(d-c)\left[\left(\int_{0}^{\frac{b-x}{b-a}} \int_{0}^{\frac{d-y}{d-c}}\left(q_{1} q_{2} t s\right)^{r_{1}} d_{q_{1}} t d_{q_{2}} s\right)^{\frac{1}{r_{1}}}\right.
\end{aligned}
$$

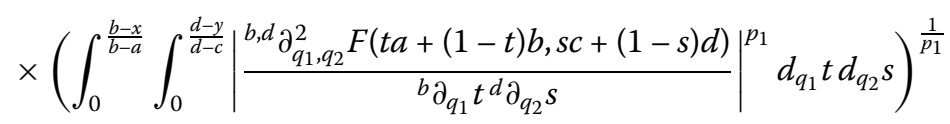

$$
\begin{aligned}
& +\left(\int_{0}^{\frac{b-x}{b-a}} \int_{\frac{d-y}{d-c}}^{1}\left(q_{1} t\left(1-q_{2} s\right)\right)^{r_{1}} d_{q_{1}} t d_{q_{2}} s\right)^{\frac{1}{r_{1}}}
\end{aligned}
$$

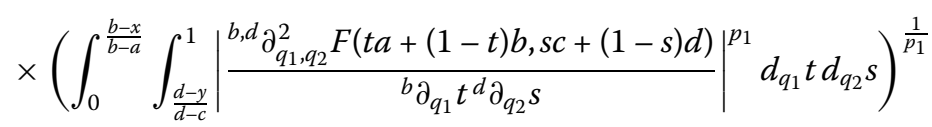

$$
\begin{aligned}
& +\left(\int_{\frac{b-x}{b-a}}^{1} \int_{0}^{\frac{d-y}{d-c}}\left(\left(1-q_{1} t\right) q_{2} s\right)^{r_{1}} d_{q_{1}} t d_{q_{2}} s\right)^{\frac{1}{r_{1}}}
\end{aligned}
$$

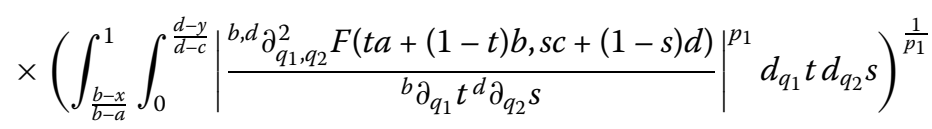

$$
\begin{aligned}
& +\left(\int_{\frac{b-x}{b-a}}^{1} \int_{\frac{d-y}{d-c}}^{1}\left(\left(1-q_{1} t\right)\left(1-q_{2} s\right)\right)^{r_{1}} d_{q_{1}} t d_{q_{2}} s\right)^{\frac{1}{r_{1}}}
\end{aligned}
$$

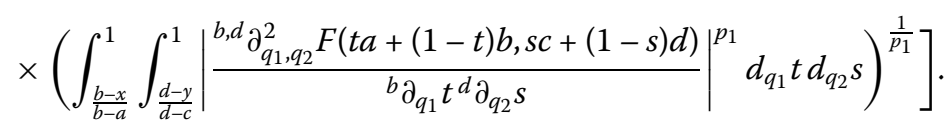

Now applying the convexity of $\left|\frac{b, d}{{ }_{1} \partial_{q_{1}, q_{2}}^{2} F(t, s)}{ }_{\partial_{q_{1}} d \partial_{q_{2}} s}\right|^{p_{1}}$, we obtain that

$$
\begin{aligned}
\left(\int_{0}^{\frac{b-x}{b-a}}\right. & \left.\int_{0}^{\frac{d-y}{d-c}}\left(q_{1} q_{2} t s\right)^{r_{1}} d_{q_{1}} t d_{q_{2}} s\right)^{\frac{1}{r_{1}}} \\
& \times\left(\left.\left.\int_{0}^{\frac{b-x}{b-a}} \int_{0}^{\frac{d-y}{d-c}}\right|^{b, d} \frac{\partial_{q_{1}, q_{2}}^{2} F(t a+(1-t) b, s c+(1-s) d)}{b \partial_{q_{1}} t d \partial_{q_{2}} s}\right|^{p_{1}} d_{q_{1}} t d_{q_{2}} s\right)^{\frac{1}{p_{1}}} \\
\leq & \left(\left(\frac{b-x}{b-a}\right)^{1+\frac{1}{r_{1}}}\left(\frac{d-y}{d-c}\right)^{1+\frac{1}{r_{1}}}\left(\frac{q_{1}}{\left[r_{1}+1\right]_{q_{1}}}\right)^{\frac{1}{r_{1}}}\left(\frac{q_{2}}{\left[r_{1}+1\right]_{q_{2}}}\right)^{\frac{1}{r_{1}}}\right) \\
& \times\left[\left|\frac{b, d \partial_{q_{1}, q_{2}}^{2} F(a, c)}{b \partial_{q_{1}} t d \partial_{q_{2}} s}\right|^{p_{1}} \frac{1}{[2]_{q_{1}}[2]_{q_{2}}}\left(\frac{b-x}{b-a}\right)^{2}\left(\frac{d-y}{d-c}\right)^{2}\right. \\
& +\left|\frac{b, d \partial_{q_{1}, q_{2}}^{2} F(a, d)}{b \partial_{q_{1}} t^{d} \partial_{q_{2}} s}\right|^{p_{1}} \frac{1}{[2]_{q_{1}}}\left(\frac{b-x}{b-a}\right)^{2}\left(\frac{d-y}{d-c}-\frac{1}{[2]_{q_{2}}}\left(\frac{d-y}{d-c}\right)^{2}\right) \\
& +\left|\frac{b, d \partial_{q_{1}, q_{2}}^{2} F(b, c)}{b \partial_{q_{1}} t^{d} \partial_{q_{2}} s}\right|^{p_{1}} \frac{1}{[2]_{q_{2}}}\left(\frac{d-y}{d-c}\right)^{2}\left(\frac{b-x}{b-a}-\frac{1}{[2]_{q_{1}}}\left(\frac{b-x}{b-a}\right)^{2}\right)
\end{aligned}
$$




$$
\begin{aligned}
& +\left|\frac{b, d}{{ }^{b} \partial_{q_{1}, q_{2}}^{2} t^{d} \partial_{q_{2}} s}\right|^{p_{1}} \\
& \left.\times\left(\frac{b-x}{b-a}-\frac{1}{[2]_{q_{1}}}\left(\frac{b-x}{b-a}\right)^{2}\right)\left(\frac{d-y}{d-c}-\frac{1}{[2]_{q_{2}}}\left(\frac{d-y}{d-c}\right)^{2}\right)\right]^{\frac{1}{p_{1}}}, \\
& \left(\int_{\frac{b-x}{b-a}}^{1} \int_{0}^{\frac{d-y}{d-c}}\left(q_{1} t\left(1-q_{2} s\right)\right)^{r_{1}} d_{q_{1}} t d_{q_{2}} s\right)^{\frac{1}{r_{1}}} \\
& \times\left(\left.\left.\int_{\frac{b-x}{b-a}}^{1} \int_{0}^{\frac{d-y}{d-c}}\right|^{b, d} \frac{\partial_{q_{1}, q_{2}}^{2} F(t a+(1-t) b, s c+(1-s) d)}{{ }^{b} \partial_{q_{1}} t^{d} \partial_{q_{2}} s}\right|^{p_{1}} d_{q_{1}} t d_{q_{2}} s\right)^{\frac{1}{p_{1}}} \\
& \leq\left(\left(\frac{d-y}{d-c}\right)^{1+\frac{1}{r_{1}}}\left(\frac{q_{2}}{\left[r_{1}+1\right]_{q_{2}}}\right)^{\frac{1}{r_{1}}}\left(\int_{\frac{b-x}{b-a}}^{1}\left(1-q_{1} t\right)^{r_{1}} d_{q_{1}} t\right)^{\frac{1}{r_{1}}}\right) \\
& \times\left[\left|\frac{{ }^{b} d \partial_{q_{1}, q_{2}}^{2} F(a, c)}{b \partial_{q_{1}} t^{d} \partial_{q_{2}} s}\right|^{p_{1}} \frac{1}{[2]_{q_{1}}[2]_{q_{2}}}\left(\frac{d-y}{d-c}\right)^{2}\left(1-\left(\frac{b-x}{b-a}\right)^{2}\right)\right. \\
& +\left|\frac{b, d}{{ }^{b} \partial_{q_{1}} t^{d} \partial_{q_{2}} s}\right|^{p_{1}} \frac{1}{[2]_{q_{1}}}\left(1-\left(\frac{b-x}{b-a}\right)^{2}\right)\left(\frac{d-y}{d-c}-\frac{1}{[2]_{q_{2}}}\left(\frac{d-y}{d-c}\right)^{2}\right) \\
& +\left|\frac{b, d}{{ }^{b} \partial_{q_{1}, q_{2}}^{2} t^{d} \partial_{q_{2}} s}\right|^{p_{1}}\left(\frac{q_{1}}{[2]_{q_{1}}}-\frac{b-x}{b-a}+\frac{1}{[2]_{q_{1}}}\left(\frac{b-x}{b-a}\right)^{2}\right)
\end{aligned}
$$

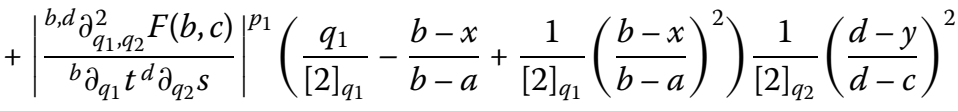

$$
\begin{aligned}
& \left.\times\left(\frac{d-y}{d-c}-\frac{1}{[2]_{q_{2}}}\left(\frac{d-y}{d-c}\right)^{2}\right)\right]^{\frac{1}{p_{1}}} \text {, } \\
& \left(\int_{0}^{\frac{b-x}{b-a}} \int_{\frac{d-y}{d-c}}^{1}\left(q_{1} t\left(1-q_{2} s\right)\right)^{r_{1}} d_{q_{1}} t d_{q_{2}} s\right)^{\frac{1}{r_{1}}}
\end{aligned}
$$

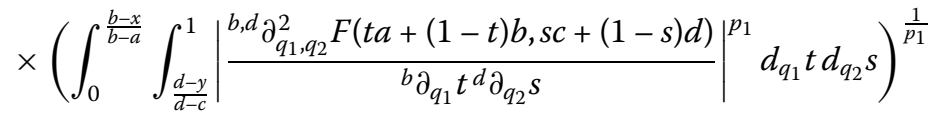

$$
\begin{aligned}
& \leq\left(\left(\frac{b-x}{b-a}\right)^{1+\frac{1}{r_{1}}}\left(\frac{q_{1}}{\left[r_{1}+1\right]_{q_{1}}}\right)^{\frac{1}{r_{1}}}\left(\int_{\frac{d-y}{d-c}}^{1}\left(1-q_{2} s\right)^{r_{1}} d_{q_{2}} s\right)^{\frac{1}{r_{1}}}\right) \\
& \times\left[\left|\frac{b, d}{{ }^{b} \partial_{q_{1}, q_{2}}^{2} F(a, c)}\right|^{{ }^{2} \partial_{q_{1}} t^{d} \partial_{q_{2}} s} \frac{1}{[2]_{q_{1}}[2]_{q_{2}}}\left(\frac{b-x}{b-a}\right)^{2}\left(1-\left(\frac{d-y}{d-c}\right)^{2}\right)\right. \\
& +\left|\frac{b, d}{{ }^{b} \partial_{q_{1}} t^{d} \partial_{q_{2}} s}\right|^{p_{1}} F(a, d) \frac{1}{[2]_{q_{1}}}\left(\frac{b-x}{b-a}\right)^{2}\left(\frac{q_{2}}{[2]_{q_{2}}}-\frac{d-y}{d-c}+\frac{1}{[2]_{q_{2}}}\left(\frac{d-y}{d-c}\right)^{2}\right) \\
& +\left|\frac{b, d}{{ }^{b} \partial_{q_{1}, q_{2}}^{2} t^{d} \partial_{q_{2}} s}\right|^{p_{1}} \frac{1}{[2]_{q_{2}}}\left(1-\left(\frac{d-y}{d-c}\right)^{2}\right)\left(\frac{b-x}{b-a}-\frac{1}{[2]_{q_{1}}}\left(\frac{b-x}{b-a}\right)^{2}\right) \\
& +\left.\left|\frac{b, d}{{ }^{b} \partial_{q_{1}, q_{2}}^{2} F(b, d)}\right|^{p_{q_{1}} \partial_{q_{2}} s}\right|^{p_{1}}\left(\frac{b-x}{b-a}-\frac{1}{[2]_{q_{1}}}\left(\frac{b-x}{b-a}\right)^{2}\right) \\
& \left.\times\left(\frac{q_{2}}{[2]_{q_{2}}}-\frac{d-y}{d-c}+\frac{1}{[2]_{q_{2}}}\left(\frac{d-y}{d-c}\right)^{2}\right)\right]^{\frac{1}{p_{1}}},
\end{aligned}
$$




$$
\begin{aligned}
& \left(\int_{\frac{b-x}{b-a}}^{1} \int_{\frac{d-y}{d-c}}^{1}\left(\left(1-q_{1} t\right)\left(1-q_{2} s\right)\right)^{r_{1}} d_{q_{1}} t d_{q_{2}} s\right)^{\frac{1}{r_{1}}}
\end{aligned}
$$

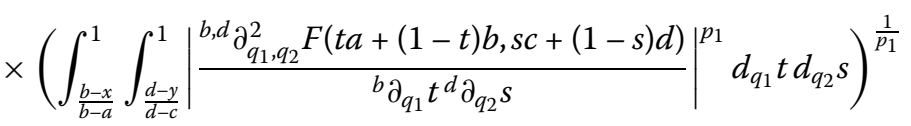

$$
\begin{aligned}
& \leq\left(\left(\int_{\frac{b-x}{b-a}}^{1}\left(1-q_{1} t\right)^{r_{1}} d_{q_{1}} t\right)^{\frac{1}{r_{1}}}\left(\int_{\frac{d-y}{d-c}}^{1}\left(1-q_{2} s\right)^{r_{1}} d_{q_{2}} s\right)^{\frac{1}{r_{1}}}\right)
\end{aligned}
$$

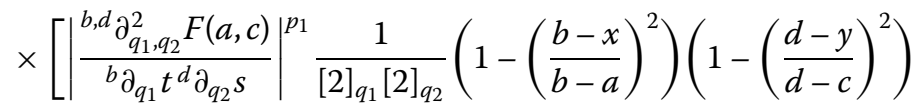

$$
\begin{aligned}
& +\left|\frac{{ }^{b, d} \partial_{q_{1}, q_{2}}^{2} F(a, d)}{{ }^{b} \partial_{q_{1}} t^{d} \partial_{q_{2}} s}\right|^{p_{1}} \frac{1}{[2]_{q_{1}}}\left(1-\left(\frac{b-x}{b-a}\right)^{2}\right)\left(\frac{q_{2}}{[2]_{q_{2}}}-\frac{d-y}{d-c}+\frac{1}{[2]_{q_{2}}}\left(\frac{d-y}{d-c}\right)^{2}\right) \\
& +\left|\frac{b, d}{{ }^{b} \partial_{q_{1}} t^{d} \partial_{q_{2}} s}\right|^{p_{1}} \frac{1}{[2]_{q_{2}}}\left(1-\left(\frac{d-y}{d-c}\right)^{2}\right)\left(\frac{q_{1}}{[2]_{q_{1}}}-\frac{b-x}{b-a}+\frac{1}{[2]_{q_{1}}}\left(\frac{b-x}{b-a}\right)^{2}\right) \\
& +\left|\frac{b, d}{{ }^{b} \partial_{q_{1}, q_{2}}^{2} t^{d} \partial_{q_{2}} s}\right|^{p_{1}}\left(\frac{q_{1}}{[2]_{q_{1}}}-\frac{b-x}{b-a}+\frac{1}{[2]_{q_{1}}}\left(\frac{b-x}{b-a}\right)^{2}\right) \\
& \left.\times\left(\frac{q_{2}}{[2]_{q_{2}}}-\frac{d-y}{d-c}+\frac{1}{[2]_{q_{2}}}\left(\frac{d-y}{d-c}\right)^{2}\right)\right]^{\frac{1}{p_{1}}} \text {. }
\end{aligned}
$$

From (4.13)-(4.17) we get the desired inequality, and the proof is accomplished.

\section{Some particular cases}

In this section, we present some particular cases of the results given in Sect. 4.

Remark 1 In Theorem 5,

(i) By taking $p_{1}=1$ and $\left|\frac{b, d \partial_{q_{1}, q_{2}}^{2} F(t, s)}{b \partial_{q_{1}} t t_{q_{2} s}}\right| \leq M$, we have the following new Ostrowski-type inequality:

$$
\begin{aligned}
& \mid \frac{1}{(b-a)(d-c)} \int_{a}^{b} \int_{c}^{d} F(t, s)^{b} d_{q_{1}} t^{d} d_{q_{2}} s-\frac{1}{b-a} \int_{a}^{b} F(t, y)^{b} d_{q_{1}} t \\
& \quad-\frac{1}{d-c} \int_{c}^{d} F(x, s)^{d} d_{q_{2}} s+F(x, y) \mid \\
& \leq \frac{M}{[2]_{q_{1}}[2]_{q_{2}}}\left[q_{1}(x-a)^{2}+\left(1-q_{1}\right)(x-a)(b-a)+q_{1}(b-x)^{2}\right] \\
& \quad \times\left[q_{2}(y-a)^{2}+\left(1-q_{2}\right)(y-c)(d-c)+q_{2}(d-y)^{2}\right] .
\end{aligned}
$$

Particularly, taking the limit as $q_{1}, q_{2} \rightarrow 1^{-}$in (5.1), we reduce inequality (5.1) to (1.4).

(ii) By taking $x=\frac{a+q_{1} b}{[2]_{1}}$ and $y=\frac{c+q_{2} d}{[2]_{q_{2}}}$ we obtain the following new midpoint inequality:

$$
\begin{aligned}
& \mid \frac{1}{(b-a)(d-c)} \int_{a}^{b} \int_{c}^{d} F(t, s)^{b} d_{q_{1}} t^{d} d_{q_{2}} s-\frac{1}{b-a} \int_{a}^{b} F\left(t, \frac{c+q_{2} d}{[2]_{q_{2}}}\right){ }^{b} d_{q_{1}} t \\
& \quad-\frac{1}{d-c} \int_{c}^{d} F\left(\frac{a+q_{1} b}{[2]_{q_{1}}}, s\right){ }^{d} d_{q_{2}} s+F\left(\frac{a+q_{1} b}{[2]_{q_{1}}}, \frac{c+q_{2} d}{[2]_{q_{2}}}\right) \mid \\
& \leq(b-a)(d-c)\left[B_{1}^{1-\frac{1}{p_{1}}}\left(q_{1}\right) B_{1}^{1-\frac{1}{p_{1}}}\left(q_{2}\right)\right.
\end{aligned}
$$




$$
\begin{aligned}
& \times\left\{B_{2}\left(q_{1}\right)\left(B_{2}\left(q_{2}\right)\left|\frac{\partial_{q_{1}, q_{2}}^{2} F(a, c)}{b \partial_{q_{1}} t^{d} \partial_{q_{2}} s}\right|^{p_{1}}+B_{3}\left(q_{2}\right)\left|\frac{\partial_{q_{1}, q_{2}} F(a, d)}{b \partial_{q_{1}} t^{d} \partial_{q_{2}} s}\right|^{p_{1}}\right)\right. \\
& \left.+B_{3}\left(q_{1}\right)\left(B_{2}\left(q_{2}\right)\left|\frac{b, d}{\partial_{q_{1}, q_{2}}^{2} F(b, c)}\right|^{b_{q_{1}} t^{d} \partial_{q_{2}} s}+B_{3}\left(q_{2}\right)\left|\frac{p^{b, d} \partial_{q_{1}, q_{2}}^{2} F(b, d)}{b \partial_{q_{1}} t^{d} \partial_{q_{2}} s}\right|^{p_{1}}\right)\right\}^{\frac{1}{p_{1}}} \\
& +B_{1}^{1-\frac{1}{p_{1}}}\left(q_{1}\right) B_{1}^{1-\frac{1}{p_{1}}}\left(q_{2}\right) \\
& \times\left\{B_{2}\left(q_{1}\right)\left(B_{4}\left(q_{2}\right)\left|\frac{b, d}{\partial_{q_{1}, q_{2}}^{2} F(a, c)} \frac{p_{1}}{b \partial_{q_{1}} t^{d} \partial_{q_{2}} s}\right|^{p_{1}}+B_{5}\left(q_{2}\right)\left|\frac{\partial_{q_{1}, q_{2}}^{2} F(a, d)}{b \partial_{q_{1}} t^{d} \partial_{q_{2}} s}\right|^{p_{1}}\right)\right. \\
& \left.+B_{3}\left(q_{1}\right)\left(B_{4}\left(q_{2}\right)\left|\frac{b, d}{\partial_{q_{1}, q_{2}}^{2} F(b, c)}\right|^{b_{q_{1}} t^{d} \partial_{q_{2}} s}+B_{5}\left(q_{2}\right)\left|\frac{p_{1}, d \partial_{q_{1}, q_{2}}^{2} F(b, d)}{b \partial_{q_{1}} t^{d} \partial_{q_{2}} s}\right|^{p_{1}}\right)\right\}^{\frac{1}{p_{1}}} \\
& +B_{1}^{1-\frac{1}{p_{1}}}\left(q_{1}\right) B_{1}^{1-\frac{1}{p_{1}}}\left(q_{2}\right) \\
& \times\left\{B_{4}\left(q_{1}\right)\left(B_{2}\left(q_{2}\right)\left|\frac{b, d \partial_{q_{1}, q_{2}}^{2} F(a, c)}{b \partial_{q_{1}} t^{d} \partial_{q_{2}} s}\right|^{p_{1}}+B_{3}\left(q_{2}\right)\left|\frac{\partial_{q_{1}, q_{2}} F(a, d)}{b \partial_{q_{1}} t^{d} \partial_{q_{2}} s}\right|^{p_{1}}\right)\right.
\end{aligned}
$$

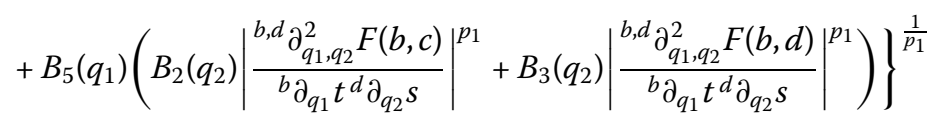

$$
\begin{aligned}
& +B_{1}^{1-\frac{1}{p_{1}}}\left(q_{1}\right) B_{1}^{1-\frac{1}{p_{1}}}\left(q_{2}\right) \\
& \times\left\{B_{4}\left(q_{1}\right)\left(B_{4}\left(q_{2}\right)\left|\frac{b^{, d} \partial_{q_{1}, q_{2}}^{2} F(a, c)}{b \partial_{q_{1}} t^{d} \partial_{q_{2}} s}\right|^{p_{1}}+B_{5}\left(q_{2}\right)\left|\frac{\partial_{q_{1}, q_{2}} F(a, d)}{b \partial_{q_{1}} t^{d} \partial_{q_{2}} s}\right|^{p_{1}}\right)\right. \\
& \left.\left.+B_{5}\left(q_{1}\right)\left(B_{4}\left(q_{2}\right)\left|\frac{b, d}{b_{q_{1}, q_{2}}^{2} F(b, c)}\right|^{p_{1} \partial_{q_{1}} t^{d} \partial_{q_{2}} s}+B_{5}\left(q_{2}\right)\left|\frac{b, d}{{ }^{b} \partial_{q_{1}, q_{2}}^{2} t^{d} \partial_{q_{2}} s}\right|^{p_{1}}\right)\right\}^{\frac{1}{p_{1}}}\right],
\end{aligned}
$$

where

$$
\begin{aligned}
& B_{1}(q)=\frac{q}{[2]_{q}^{3}}, \quad B_{2}(q)=\frac{q}{[2]_{q}^{3}[3]_{q}}, \quad B_{3}(q)=\frac{q^{2}}{[2]_{q}^{2}[3]_{q}} \\
& B_{4}(q)=\frac{2 q}{[2]_{q}^{3}[3]_{q}}, \quad B_{5}(q)=\frac{-q+q^{2}+q^{3}}{[2]_{q}^{3}[3]_{q}}
\end{aligned}
$$

and $q_{1}, q_{2} \in(0,1)$. Particularly, taking the limit as $q_{1}, q_{2} \rightarrow 1^{-}$in (5.2), we reduce inequality (5.2) to [37, Theorem 2, inequality (2.4)]

$$
\begin{aligned}
& \mid \frac{1}{(b-a)(d-c)} \int_{a}^{b} \int_{c}^{d} F(t, s) d t d s-\frac{1}{b-a} \int_{a}^{b} F\left(t, \frac{c+d}{2}\right) d t \\
& \quad-\frac{1}{d-c} \int_{c}^{d} F\left(\frac{a+b}{2}, s\right) d s+F\left(\frac{a+b}{2}, \frac{c+d}{2}\right) \mid \\
& \leq \frac{(b-a)(d-c)}{64}\left(\frac{2}{3}\right)^{\frac{2}{p_{1}}} \\
& \quad \times\left\{\left[\frac{\frac{\partial^{2} F(a, c)}{\partial s \partial t}+2 \frac{\partial^{2} F(a, d)}{\partial s \partial t}+2 \frac{\partial^{2} F(b, c)}{\partial s \partial t}+4 \frac{\partial^{2} F(b, d)}{\partial s \partial t}}{4}\right]^{\frac{1}{p_{1}}}\right. \\
& +\left[\frac{\left.2 \frac{\partial^{2} F(a, c)}{\partial s \partial t}+\frac{\partial^{2} F(a, d)}{\partial s \partial t}+4 \frac{\partial^{2} F(b, c)}{\partial s \partial t}+2 \frac{\partial^{2} F(b, d)}{\partial s \partial t}\right]^{\frac{1}{p_{1}}}}{4}\right.
\end{aligned}
$$




$$
\begin{aligned}
& +\left[\frac{2 \frac{\partial^{2} F(a, c)}{\partial s \partial t}+4 \frac{\partial^{2} F(a, d)}{\partial s \partial t}+\frac{\partial^{2} F(b, c)}{\partial s \partial t}+2 \frac{\partial^{2} F(b, d)}{\partial s \partial t}}{4}\right]^{\frac{1}{p_{1}}} \\
& \left.+\left[\frac{4 \frac{\partial^{2} F(a, c)}{\partial s \partial t}+2 \frac{\partial^{2} F(a, d)}{\partial s \partial t}+2 \frac{\partial^{2} F(b, c)}{\partial s \partial t}+\frac{\partial^{2} F(b, d)}{\partial s \partial t}}{4}\right]^{\frac{1}{p_{1}}}\right\} .
\end{aligned}
$$

(iii) By taking $p_{1}=1$ we have the following inequality:

$$
\begin{aligned}
& \mid \frac{1}{(b-a)(d-c)} \int_{a}^{b} \int_{c}^{d} F(t, s)^{b} d_{q_{1}} t^{d} d_{q_{2}} s-\frac{1}{b-a} \int_{a}^{b} F(t, y)^{b} d_{q_{1}} x \\
& -\frac{1}{d-c} \int_{c}^{d} F(x, s)^{d} d_{q_{2}} s+F(x, y) \\
& \leq(b-a)(d-c)\left[| \frac { b , d } { \partial _ { q _ { 1 } , q _ { 2 } } ^ { 2 } F ( a , c ) } | \left\{\left(A_{2}\left(a, b, q_{1}, x\right)+A_{5}\left(a, b, q_{1}, x\right)\right)\right.\right. \\
& \left.\left.\times\left(A_{2}\left(c, d, q_{2}, y\right)+\left(A_{5}\left(c, d, q_{2}, y\right)\right)\right)\right\}\right]
\end{aligned}
$$

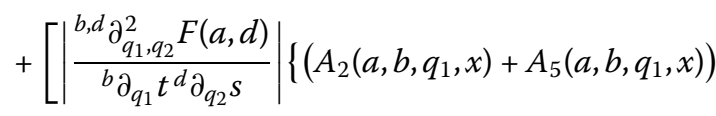

$$
\begin{aligned}
& \left.\left.\times\left(A_{3}\left(c, d, q_{2}, y\right)+\left(A_{6}\left(c, d, q_{2}, y\right)\right)\right)\right\}\right]
\end{aligned}
$$

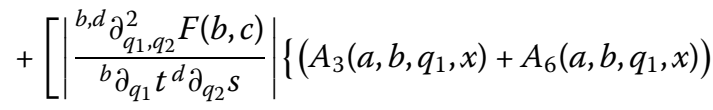

$$
\begin{aligned}
& \left.\left.\times\left(A_{2}\left(c, d, q_{2}, y\right)+\left(A_{5}\left(c, d, q_{2}, y\right)\right)\right)\right\}\right] \\
& +\left[| \frac { b , d } { \partial _ { q _ { 1 } , q _ { 2 } } ^ { 2 } F ( b , d ) } | \left\{\left(A_{3}\left(a, b, q_{1}, x\right)+A_{6}\left(a, b, q_{1}, x\right)\right)\right.\right. \\
& \left.\left.\times\left(A_{3}\left(c, d, q_{2}, y\right)+\left(A_{6}\left(c, d, q_{2}, y\right)\right)\right)\right\}\right] \text {. }
\end{aligned}
$$

Particularly, taking the limit as $q_{1}, q_{2} \rightarrow 1^{-}$in (5.4), we reduce inequality (5.4) reduces to [40, Theorem 2].

\section{Remark 2 Consider Theorem 6.}

(i) If $\left|\frac{b, d \partial_{q_{1}, q_{2}}^{2} F(t, s)}{b \partial_{q_{1}} t^{d} \partial_{q_{2}} s}\right| \leq M$, then as $q_{1}, q_{2} \rightarrow 1^{-}$, we obtain inequality (1.5).

(ii) Taking $x=\frac{a+q_{1} b}{[2] q_{1}}$ and $y=\frac{c+q_{2} d}{[2] q_{2}}$, we obtain the following new midpoint inequality:

$$
\begin{aligned}
& \mid \frac{1}{(b-a)(d-c)} \int_{a}^{b} \int_{c}^{d} F(t, s)^{b} d_{q_{1}} t^{d} d_{q_{2}} s-\frac{1}{b-a} \int_{a}^{b} F\left(t, \frac{c+q_{2} d}{[2]_{q_{2}}}\right){ }^{b} d_{q_{1}} t \\
& \quad-\frac{1}{d-c} \int_{c}^{d} F\left(\frac{a+q_{1} b}{[2]_{q_{1}}}, s\right){ }^{d} d_{q_{2}} s+F\left(\frac{a+q_{1} b}{[2]_{q_{1}}}, \frac{c+q_{2} d}{[2]_{q_{2}}}\right) \mid \\
& \leq(b-a)(d-c) \\
& \times\left[\left(\left(\frac{1}{[2]_{q_{1}}}\right)^{1+\frac{1}{r_{1}}}\left(\frac{1}{[2]_{q_{2}}}\right)^{1+\frac{1}{r_{1}}}\left(\frac{q_{1}}{\left[r_{1}+1\right]_{q_{1}}}\right)^{\frac{1}{r_{1}}}\left(\frac{q_{2}}{\left[r_{1}+1\right]_{q_{2}}}\right)^{\frac{1}{r_{1}}}\right)\right.
\end{aligned}
$$




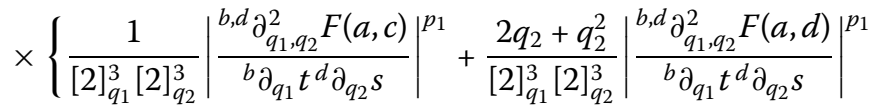

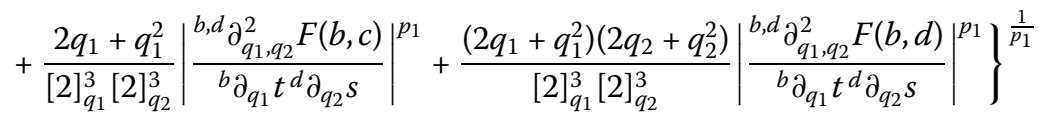

$$
\begin{aligned}
& +\left(\left(\frac{1}{[2]_{q_{2}}}\right)^{1+\frac{1}{r_{1}}}\left(\frac{q_{2}}{\left[r_{1}+1\right]_{q_{2}}}\right)^{\frac{1}{r_{1}}}\left(\int_{\frac{1}{[2]_{q_{1}}}}^{1}\left(1-q_{1} t\right)^{r_{1}} d_{q_{1}} t\right)^{\frac{1}{r_{1}}}\right)
\end{aligned}
$$

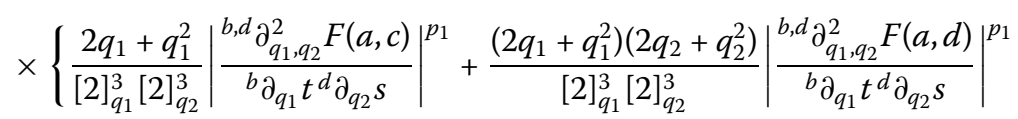

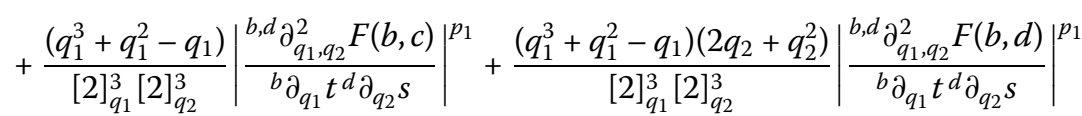

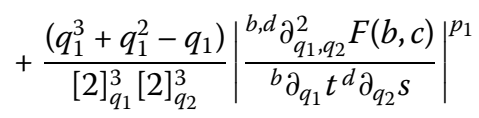

$$
\begin{aligned}
& \left.+\frac{\left(q_{1}^{3}+q_{1}^{2}-q_{1}\right)\left(2 q_{2}+q_{2}^{2}\right)}{[2]_{q_{1}}^{3}[2]_{q_{2}}^{3}}\left|\frac{{ }^{b, d} \partial_{q_{1}, q_{2}}^{2} F(b, d)}{b \partial_{q_{1}} t^{d} \partial_{q_{2}} s}\right|^{p_{1}}\right\}^{\frac{1}{p_{1}}} \\
& +\left(\left(\frac{1}{[2]_{q_{1}}}\right)^{1+\frac{1}{r_{1}}}\left(\frac{q_{1}}{\left[r_{1}+1\right]_{q_{1}}}\right)^{\frac{1}{r_{1}}}\left(\int_{\frac{1}{[2] q_{2}}}^{1}\left(1-q_{2} s\right)^{r_{1}} d_{q_{2}} s\right)^{\frac{1}{r_{1}}}\right)
\end{aligned}
$$

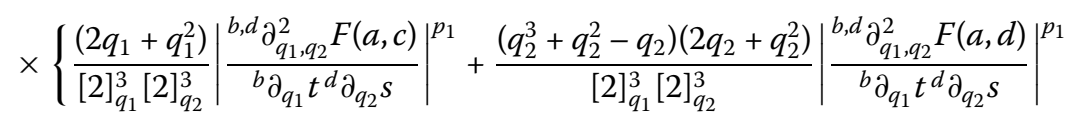

$$
\begin{aligned}
& +\left.\frac{\left(2 q_{1}+q_{1}^{2}\right)\left(2 q_{2}+q_{2}^{2}\right)}{[2]_{q_{1}}^{3}[2]_{q_{2}}^{3}}\left|\frac{b, d}{\partial_{q_{1}, q_{2}}^{2} F(b, c)}\right|^{b_{q_{1}} t^{d} \partial_{q_{2}} s}\right|^{p_{1}} \\
& \left.+\frac{\left(q_{2}^{3}+q_{2}^{2}-q_{2}\right)\left(2 q_{2}+q_{2}^{2}\right)}{[2]_{q_{1}}^{3}[2]_{q_{2}}^{3}}\left|\frac{\partial_{q_{1}, q_{2}}^{2} F(b, d)}{b \partial_{q_{1}} t^{d} \partial_{q_{2}} s}\right|^{p_{1}}\right\}^{\frac{1}{p_{1}}} \\
& +\left(\left(\int_{\frac{1}{[2]_{q_{1}}}}^{1}\left(1-q_{1} t\right)^{r_{1}} d_{q_{1}} t\right)^{\frac{1}{r_{1}}}\left(\int_{\frac{1}{[2]_{q_{2}}}}^{1}\left(1-q_{2} s\right)^{r_{1}} d_{q_{2}} s\right)^{\frac{1}{r_{1}}}\right)
\end{aligned}
$$

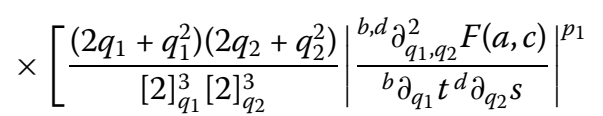

$$
\begin{aligned}
& +\left.\frac{\left(2 q_{1}+q_{1}^{2}\right)\left(q_{2}^{3}+q_{2}^{2}-q_{2}\right)}{[2]_{q_{1}}^{3}[2]_{q_{2}}^{3}}\left|\frac{b, d}{\partial_{q_{1}, q_{2}}^{2} F(a, d)}\right|^{b \partial_{q_{1}} t^{d} \partial_{q_{2}} s}\right|^{p_{1}}
\end{aligned}
$$

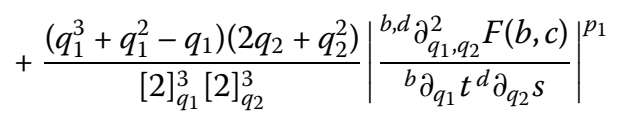

$$
\begin{aligned}
& \left.+\left.\frac{\left(q_{1}^{3}+q_{1}^{2}-q_{1}\right)\left(q_{2}^{3}+q_{2}^{2}-q_{2}\right)}{[2]_{q_{1}}^{3}[2]_{q_{2}}^{3}}\left|\frac{b, d}{\partial_{q_{1}, q_{2}}^{2} F(b, d)}\right|^{{ }^{p_{1}} \partial_{q_{1}} t^{d} \partial_{q_{2}} s}\right|^{\frac{1}{p_{1}}}\right] \text {. }
\end{aligned}
$$

\section{Conclusion}

In this research, we proved some new quantum Ostrowski-type inequalities for $q_{1} q_{2}$ differentiable coordinated convex functions using the $q_{1} q_{2}$-integrals. We also showed that the results proved in this research transformed into some new and known inequalities by considering the limits as $q_{1}, q_{2} \rightarrow 1^{-}$in the main results. It is interesting that the forth- 
coming researchers can offer similar inequalities for different kinds of convexities in their future work.

\author{
Acknowledgements \\ The authors would like to express their sincere thanks to the editor and the anonymous reviewers for their helpful \\ comments and suggestions.
}

\title{
Funding
}

The work was supported by the Natural Science Foundation of China (Grant Nos. 61673169, 11301127, 11701176, $11626101,11601485,11971241)$.

Availability of data and materials

Not applicable.

\section{Competing interests}

The authors declare that they have no competing interests.

\section{Authors' contributions}

All authors contributed equally to the writing of this paper. All authors read and approved the final manuscript.

\section{Author details}

${ }^{1}$ Jiangsu Key Laboratory for NSLSCS, School of Mathematical Sciences, Nanjing Normal University, Nanjing, China.

${ }^{2}$ Department of Mathematics, Huzhou University, Huzhou, China. ${ }^{3}$ Department of Mathematics, Faculty of Science and Arts, Düzce University, Düzce, Turkey. ${ }^{4}$ Department of Mathematics, Faculty of Science and Arts, University of Kahramanmaraş Sütçü İmam, Kahramanmaraş, Turkey. ${ }^{5}$ Department of Mathematics, COMSATS University Islamabad Sahiwal Campus, Sahiwal, Pakistan.

\section{Publisher's Note}

Springer Nature remains neutral with regard to jurisdictional claims in published maps and institutional affiliations.

Received: 14 August 2020 Accepted: 20 December 2020 Published online: 07 January 2021

\section{References}

1. Ernst, T.: A Comprehensive Treatment of q-Calculus. Springer, Berlin (2012)

2. Gauchman, H.: Integral inequalities in q-calculus. Comput. Math. Appl. 47(2-3), 281-300 (2004)

3. Ernst, T.: The history of Q-calculus and a new method. Department of Mathematics, Uppsala University, Sweden (2000)

4. Jackson, F.H.: On q-definite integrals. Q. J. Pure Appl. Math. 41, 193-203 (1910)

5. Agarwal, R.P.: A propos d'une note de M. Pierre Humbert. C. R. Acad. Sci. Paris, Ser. I 236(21), $2031-2032$ (1953)

6. Al-Salam, W.A.: Some fractional $q$-integrals and q-derivatives. Proc. Edinb. Math. Soc. 15(2), 135-140 (1966)

7. Alp, N., Sarıkaya, M.Z., Kunt, M., İşcan, İ.: q-Hermite Hadamard inequalities and quantum estimates for midpoint type inequalities via convex and quasi-convex functions. J. King Saud Univ., Sci. 30(2), 193-203 (2018)

8. Bermudo, S., Kórus, P., Valdés, J.N.: On q-Hermite-Hadamard inequalities for general convex functions. Acta Math Hung. 162, 364-374 (2020)

9. Khan, M.A., Mohammad, N., Nwaeze, E.R., Chu, Y.-M.: Quantum Hermite-Hadamard inequality by means of a Green function. Adv. Differ. Equ. 2020(1), 99 (2020)

10. Xu, L., Chu, Y.-M., Rashid, S., El-Deeb, A.A., Nisar, K.S.: On new unified bounds for a family of functions via fractional q-calculus theory. J. Funct. Spaces 2020, Article ID 4984612 (2020)

11. Yang, X.-Z., Farid, G., Nazeer, W., Yussouf, M., Chu, Y.-M., Dong, C.-F.: Fractional generalized Hadamard and Fejér-Hadamard inequalities for m-convex functions. AIMS Math. 5(6), 6325-6340 (2020)

12. Guo, S.-Y., Chu, Y.-M., Farid, G., Mehmood, S., Nazeer, W.: Fractional Hadamard and Fejér-Hadamard inequalities associated with exponentially (s, m)-convex functions. J. Funct. Spaces 2020, Article ID 2410385 (2020)

13. Kunt, M., İşcan, I., Alp, N., Sarıkaya, M.Z.: $(p, q)$-Hermite-Hadamard inequalities and ( $p, q)$-estimates for midpoint type inequalities via convex and quasi-convex functions. Rev. R. Acad. Cienc. Exactas Fís. Nat., Ser. A Mat. 112(4), 969-992 (2018)

14. Liu, W.-J., Zhuang, H.-F.: Some quantum estimates of Hermite-Hadamard inequalities for convex functions. J. Appl. Anal. Comput. 7(2), 501-522 (2017)

15. Noor, M.A., Noor, K.I., Awan, M.U.: Some quantum estimates for Hermite-Hadamard inequalities. Appl. Math. Comput. $251,675-679(2015)$

16. Noor, M.A., Noor, K.I., Awan, M.U.: Some quantum integral inequalities via preinvex functions. Appl. Math. Comput. 269, 242-251 (2015)

17. Noor, M.A., Awan, M.U., Noor, K.I.: Quantum Ostrowski inequalities for q-differentiable convex functions. J. Math Inequal. 10(4), 1013-1018 (2016)

18. Nwaeze, E.R., Tameru, A.M.: New parameterized quantum integral inequalities via $\eta$-quasiconvexity. Adv. Differ. Equ. 2019(1), 425 (2019)

19. Sudsutad, W., Ntouyas, S.K., Tariboon, J.: Quantum integral inequalities for convex functions. J. Math. Inequal. 9(3), 781-793 (2015)

20. Zhuang, H., Liu, W., Park, J.: Some quantum estimates of Hermite-Hadamard inequalities for quasi-convex functions. Mathematics 7(2), 152 (2019) 
21. Ostrowski, A.: Über die Absolutabweichung einer differentiebaren Funcktion von ihrem Integralmittelwert. Comment. Math. Helv. 10, 226-227 (1938)

22. Alomari, M., Darus, M., Dragomir, S.S., Cerone, P.: Ostrowski type inequalities for functions whose derivatives are s-convex in the second sense. Appl. Math. Lett. 23(9), 1071-1076 (2010)

23. Alomari, M., Darus, M.: Some Ostrowski's type inequalities for convex functions with applications. Rep. Coll. 13(2) (2010)

24. Barnett, N.S., Dragomir, S.S.: An Ostrowski type inequality for double integrals and applications for cubature formulae. RGMIA research report collection 1(1), (2001)

25. Cerone, P., Dragomir, S.S.: Ostrowski type inequalities for functions whose derivatives satisfy certain convexity assumptions. Demonstr. Math. 37(2), 299-308 (2004)

26. Dragomir, S.S., Sofo, A.: Ostrowski type inequalities for functions whose derivatives are convex. In: Proceeding of the 4th International Conference on Modelling and Simulation (2002)

27. Dragomir, S.S., Barnett, N.S., Cerone, P.: An $n$-dimensional version of Ostrowski's inequality for mappings of the Hölder type. RGMIA Research Report Collection 2(2) (1999)

28. Latif, M.A. Dragomir, S., Matouk, A.: New inequalities of Ostrowski type for co-ordinated convex functions via fractional integrals. J. Fract. Calc. Appl. 2(1), 1-15 (2012)

29. Pachpatte, B.: On an inequality of Ostrowski type in three independent variables. J. Math. Anal. Appl. 249(2), 583-591 (2000)

30. Pachpatte, B.: On a new Ostrowski type inequality in two independent variables. Tamkang J. Math. 32(1), 45-49 (2001)

31. Pachpatte, B.: A new Ostrowski type inequality for double integrals. Soochow J. Math. 32(2), 317 (2006)

32. Sarikaya, M.: On the Ostrowski type integral inequality. Acta Math. Univ. Comen. 79(1), 129-134 (2010)

33. Latif, M.A., Hussain, S., Dragomir, S.S.: New Ostrowski type inequalities for co-ordinated convex functions. TJMM 4(2), 125-136 (2012)

34. Kac, V., Cheung, P.: Quantum Calculus. Springer, Berlin (2001)

35. Tariboon, J., Ntouyas, S.K.: Quantum calculus on finite intervals and applications to impulsive difference equations. Adv. Differ. Equ. 2013(1), 282 (2013)

36. Tariboon, J., Ntouyas, S.K.: Quantum integral inequalities on finite intervals. J. Inequal. Appl. 2014(1), 121 (2014)

37. Latif, M., Dragomir, S.S., Momoniat, E.: Some $q$-analogues of Hermite-Hadamard inequality of functions of two variables on finite rectangles in the plane. J. King Saud Univ., Sci. 29(3), 263-273 (2017)

38. Budak, H., Ali, M.A., Tarhanaci, M.: Some new quantum Hermite-Hadamard-like inequalities for coordinated convex functions. J. Optim. Theory Appl. 186(3), 899-910 (2020)

39. Kalsoom, H., Idrees, M., Baleanu, D., Chu, Y.-M.: New estimates of $q_{1} q_{2}$-Ostrowski-type inequalities within a class of n-ploynomial prevexity of functions. J. Funct. Spaces 2020, Article ID 3720798 (2020)

40. Latif, M., Dragomir, S.S.: On some new inequalities for differentiable co-ordinated convex functions. J. Inequal. Appl. 2012(1), 28 (2012)

\section{Submit your manuscript to a SpringerOpen ${ }^{\circ}$ journal and benefit from:}

- Convenient online submission

- Rigorous peer review

- Open access: articles freely available online

- High visibility within the field

- Retaining the copyright to your article

Submit your next manuscript at $\gg$ springeropen.com 